\title{
In-Source Laser Spectroscopy with the Laser Ion Source and Trap: First Direct Study of the Ground-State Properties of ${ }^{217,219}$ Po
}

\author{
D. A. Fink,${ }^{1,2,3,}{ }^{*}$ T. E. Cocolios, ${ }^{4,5}$ A. N. Andreyev, ${ }^{6,7}$ S. Antalic, ${ }^{8}$ A. E. Barzakh, ${ }^{9}$ B. Bastin, ${ }^{10}$ D. V. Fedorov, ${ }^{9}$ \\ V. N. Fedosseev, ${ }^{1}$ K. T. Flanagan, ${ }^{4}$ L. Ghys, ${ }^{11,12}$ A. Gottberg, ${ }^{1,13}$ M. Huyse, ${ }^{11}$ N. Imai,${ }^{14}$ T. Kron, ${ }^{15}$ N. Lecesne, ${ }^{10}$ \\ K. M. Lynch, ${ }^{4,5,11}$ B. A. Marsh, ${ }^{1}$ D. Pauwels, ${ }^{12}$ E. Rapisarda,${ }^{5}$ S. D. Richter, ${ }^{15}$ R. E. Rossel, ${ }^{1,15}$ S. Rothe,${ }^{11}{ }^{15}$ \\ M. D. Seliverstov, ${ }^{6,9}$ A. M. Sjödin,${ }^{10}$ C. Van Beveren, ${ }^{11}$ P. Van Duppen, ${ }^{11}$ and K. D. A. Wendt ${ }^{15}$ \\ ${ }^{1}$ EN Department, CERN, CH-1211 Geneva, Switzerland \\ ${ }^{2}$ Max-Planck-Institut für Kernphysik, DE-69117 Heidelberg, Germany \\ ${ }^{3}$ Fakultät für Physik und Astronomie, Ruprecht-Karls Universität, DE-69120 Heidelberg, Germany \\ ${ }^{4}$ School of Physics and Astronomy, The University of Manchester, Manchester M13 9PL, United Kingdom \\ ${ }^{5}$ ISOLDE, PH Department, CERN, CH-1211 Geneva, Switzerland \\ ${ }^{6}$ Department of Physics, The University of York, York YO10 5DD, United Kingdom \\ ${ }^{7}$ Advanced Science Research Centre, Japan Atomic Energy Agency, Tokai-mura, Ibaraki 319-1195, Japan \\ ${ }^{8}$ Department of Nuclear Physics and Biophysics, Comenius University, SK-842 48 Bratislava, Slovakia \\ ${ }^{9}$ Petersburg Nuclear Physics Institute, NRC Kurchatov Institute, 188300 Gatchina, Russia \\ ${ }^{10}$ Grand Accélérateur National d'Ions Lourds, FR-14076 Caen, France \\ ${ }^{11} \mathrm{KU}$ Leuven, Instituut voor Kern- en Stralingsfysica, BE-3001 Leuven, Belgium \\ ${ }^{12}$ Belgian Nuclear Research Centre SCK•CEN, BE-2400 Mol, Belgium \\ ${ }^{13}$ Instituto de Estructura de la Materia, CSIC, ES-28006 Madrid, Spain \\ ${ }^{14}$ High Energy Accelerator Research Organization (KEK), Tsukuba, Ibaraki 305-0801, Japan \\ ${ }^{15}$ Institut für Physik, Johannes Gutenberg-Universität Mainz, DE-55128 Mainz, Germany
}

(Received 22 October 2014; published 20 February 2015)

\begin{abstract}
A Laser Ion Source and Trap (LIST) for a thick-target, isotope-separation on-line facility has been implemented at CERN ISOLDE for the production of pure, laser-ionized, radioactive ion beams. It offers two modes of operation, either as an ion guide, which performs similarly to the standard ISOLDE resonance ionization laser ion source (RILIS), or as a more selective ion source, where surface-ionized ions from the hot ion-source cavity are repelled by an electrode, while laser ionization is done within a radiofrequency quadrupole ion guide. The first physics application of the LIST enables the suppression of francium contamination in ion beams of neutron-rich polonium isotopes at ISOLDE by more than 1000 with a reduction in laser-ionization efficiency of only 20. Resonance ionization spectroscopy is performed directly inside the LIST device, allowing the study of the hyperfine structure and isotope shift of ${ }^{217}$ Po for the first time. Nuclear decay spectroscopy of ${ }^{219}$ Po is performed for the first time, revealing its half-life, $\alpha$ to- $\beta$-decay branching ratio, and $\alpha$-particle energy. This experiment demonstrates the applicability of the LIST at radioactive ion-beam facilities for the production and study of pure beams of exotic isotopes.
\end{abstract}

DOI: 10.1103/PhysRevX.5.011018

\section{INTRODUCTION}

The study of radioactive neutron-rich nuclei in the lead region, where $Z=82$ is a nuclear magic number, is an experimental challenge. This region is inaccessible by fusion-evaporation reactions with beams of stable nuclei, while the cross section for fragmentation and spallation of

\footnotetext{
*Present address: BE Department, CERN, CH-1211 Geneva, Switzerland.

Published by the American Physical Society under the terms of the Creative Commons Attribution 3.0 License. Further distribution of this work must maintain attribution to the author(s) and the published article's title, journal citation, and DOI.
}

Subject Areas: Atomic and Molecular Physics,

Nuclear Physics heavier nuclei is distributed over a wide range of isotopes, leaving those of interest hidden by more abundant contaminants. A great effort has nonetheless been invested in recent years to study nuclei far beyond the nuclear magic number $N=126$, due to their importance for the shell evolution away from nuclear stability, for astronuclear processes, and for the search for physics beyond the standard model in octupole-deformed atomic nuclei [1-5].

Because of advances at on-line radioactive ion-beam facilities [6], such as ISOLDE at CERN [7], these isotopes are now routinely becoming available from proton-induced nuclear reactions with ${ }^{238} \mathrm{U}$. The reaction products are extracted from the target, ionized, separated in magnetic fields, and delivered to experiments as low-energy ion beams (up to $60 \mathrm{keV}$ ) of a chosen isotope. This approach is 
referred to as isotope separation on-line (ISOL). In many cases, the isotope-separation process does not provide sufficient purity and an unwanted isobaric contaminant may dominate the extracted ion beam, thereby limiting the feasibility of a particular experiment. The introduction of the resonance laser-ionization technique has increased the element selectivity of the ionization process [8], which has enabled a large research program to be undertaken, among others, in the lead region; see, e.g., Refs. [9-17]. Alternative production routes are found in the combination of in-flight fusion-evaporation or fragmentation facilities with a gas catcher [18-20]. Although the underlying concepts are different, the final approach of ionization or ion extraction, followed by separation, remains similar, and those facilities will face the same difficulties as ISOL facilities.

While the resonance ionization process enhances selectively the element of interest, it does not specifically reduce the other ionization mechanisms, such as surface ionization. The Resonance Ionization Laser Ion Source (RILIS) [21] at ISOLDE makes use of a standard-surface-ionsource cavity as it provides a confined space for the laser-atom interaction. However, the elements with a low ionization energy (IE) (e.g., ${ }_{87} \mathrm{Fr},{ }_{88} \mathrm{Ra}$ ) are surface ionized at the walls of the hot cavity alongside the laser-ionization process. Attempts to suppress the surface ions through the use of low-work-function cavity materials [22] or the pulsed-release technique [23] have been successfully applied in cases where only moderate selectivity enhancement is sufficient (e.g., for $215 \leq A \leq 218$ [9-13]). In the case of ${ }^{219} \mathrm{Po}$, however, the RILIS-ionized polonium beam is typically contaminated with $10^{5}$ times more francium if ionization takes place inside a surface-ion-source cavity.

The Laser Ion Source and Trap (LIST) uses an alternative approach. It geometrically decouples the volume where the laser ionization takes place from where other ionization mechanisms occur. The LIST technology, proposed first in 2003 [24], was predominantly developed at the Johannes Gutenberg-Universität Mainz, consecutively undergoing a number of adaptations to match conditions and operation at on-line facilities $[25,26]$. In a slightly modified version using a sextupole structure, it was also applied to a laser ion source coupled with a gas catcher, first at the LISOL setup of the Cyclotron Research Centre (Louvain-La-Neuve, Belgium) [27] and more recently at the IGISOL facility of the University of Jyväskylä (Finland) [28,29]. Lately, it has been successfully launched as a standard add-on unit for use with the hot-cavity RILIS at the thick-target radioactive ion-beam facilities ISOLDE [30,31] and TRIUMF-ISAC [32,33].

The new developments at ISAC have enabled the successful study of radioactive magnesium isotopes with $A<24$ in high-purity conditions $[34,35]$. These studies have demonstrated a suppression factor for contaminants of $10^{6}$ against a reduction of the beam of interest of 50 .
A similar performance is found at ISOLDE in the same region, where a suppression of $\gg 10^{4}$ against a reduction of the beam of interest of 50 is measured. In both cases, beams are extracted after the irradiation of a light target ( $\mathrm{SiC}$ at ISAC, Ti foils at ISOLDE) with a primary proton beam. Such a combination of target and ion source cannot, however, produce the beams of interest in this discussion, for which thorium or uranium is required as the target material. Following the irradiation of such targets, much higher ion currents and radiation levels are expected, from which additional effects may arise (e.g., radiation-induced ionization, space-charge saturation).

We reported recently on the technical aspects of the first successful use of the LIST at ISOLDE for on-line production of radioisotopes from a uranium-based target with an improved laser ionization efficiency of a factor of 2.5 leading to a reduced loss of the beam of interest of only a factor of 20 [36]. We present here a performance study of the LIST for nuclei in the region of $Z>82$ and $N>126$ and its suitability for the study of nuclear ground-state properties. In particular, the list has enabled the first measurement of the hyperfine structure and isotope shift of ${ }^{217} \mathrm{Po}$ and the first nuclear decay study of ${ }^{219} \mathrm{Po}$, which are both of interest to delineate the region of octupoledeformed nuclei [5,37].

\section{EXPERIMENT}

\section{A. Production of polonium at ISOLDE}

At ISOLDE, polonium isotopes are produced by spallation and fragmentation reactions of ${ }^{238} \mathrm{U}$ when the pulsed 1.4-GeV proton beam of the CERN proton synchrotron (PS) Booster impinges on the ISOLDE uranium-carbide target. The reaction products diffuse from the porous target material and leave the target container by effusion through the transfer line and hot cavity. To leave the target assembly, the atoms must effuse along a tantalum transfer line and ionizer cavity. Resistive heating is used to maintain a temperature of $T \approx 2300 \mathrm{~K}$ to enhance the effusion process. After ionization in the LIST (as detailed in a subsequent paragraph), the reaction products are extracted as an ion beam with an energy of up to $60 \mathrm{keV}$. Most of the results presented here are obtained using the general purpose separator (GPS) with a resolving power typically $R=(m / \Delta m) \approx 2500$. The beam is then delivered to the Windmill $\alpha$-decay setup [38]. A description of this process can be found in Fig. 1 and Ref. [36].

\section{B. Resonant ionization of polonium}

Reference [39] provides a technical description of the ISOLDE RILIS setup. The three-step photoexcitation scheme for polonium is shown in Fig. 2 [40]. This scheme uses a frequency-tripled dye laser operating at $255.8 \mathrm{~nm}(P \approx 20 \mathrm{~mW})$ for the transition to the $6 p^{3}\left(4 S^{\circ}\right) 7 s^{5} S_{2}^{\circ}$ first excited state, a tunable narrow-band 


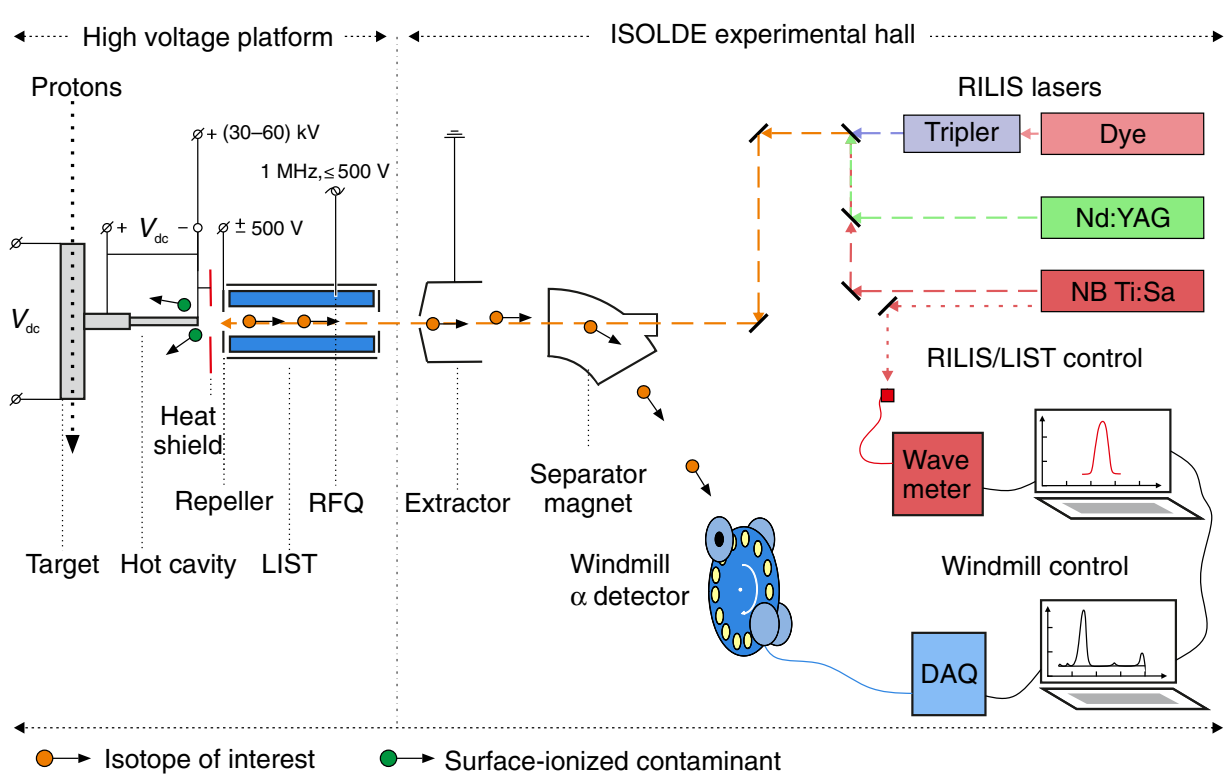

FIG. 1. An illustration of the LIST operated at the ISOLDE mass-separator facility. The LIST is attached to the target-ion-source assembly. Protons irradiate the target, and reaction products diffuse and effuse from the target into the LIST RFQ, where the atoms are ionized by the laser radiations. After extraction and acceleration by the extractor electrode at up to $60 \mathrm{kV}$, the ions are sent through the magnetic-dipole mass separator to the Windmill detection setup and data acquisition (DAQ).

(NB) Ti:sapphire laser [41] at approximately $843.4 \mathrm{~nm}$ $(P \approx 1 \mathrm{~W}$, FWHM $0.8 \mathrm{GHz})$ for the scan of the transition to the $6 p^{3}\left({ }^{4} S^{\circ}\right) 7 p^{5} P_{2}$ second excited state, and the 532-nm $(P>10 \mathrm{~W})$ second harmonic output of a neodymiumdoped yttrium-aluminum-garnet (Nd:YAG) laser for the nonresonant transition over the ionization energy (IE) into

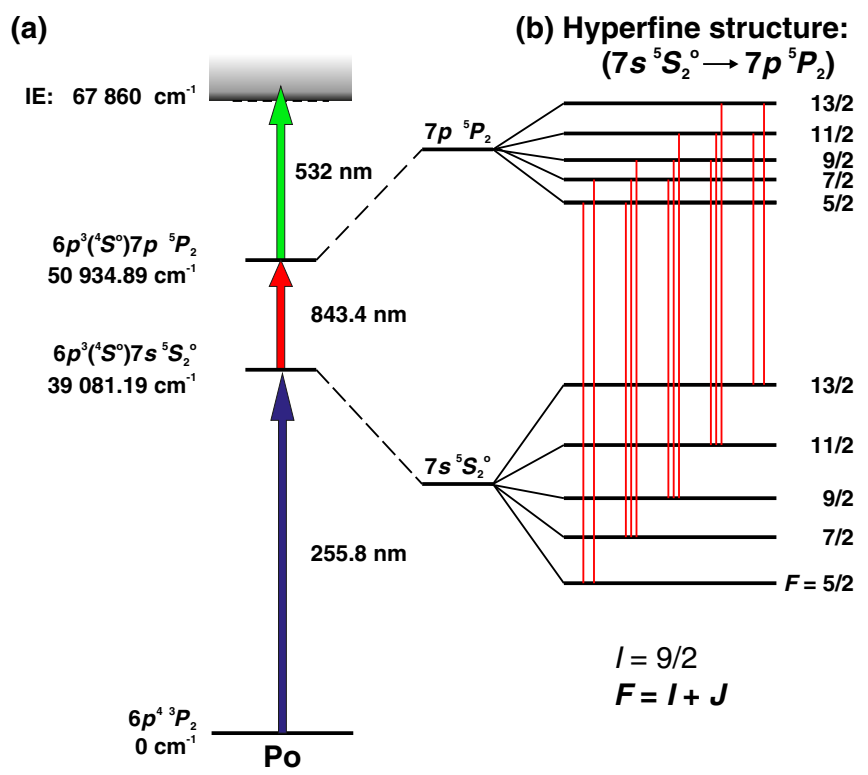

FIG. 2. (a) Three-step laser-ionization scheme for polonium. (b) Close-up of the possible hyperfine levels for the nuclear spin $I=9 / 2$ with total angular momentum $F$ and corresponding transitions (solid red lines) for the studied transition from the $7 s^{5} S_{2}^{\circ}$ state to the $7 p^{5} P_{2}$ state. The hyperfine structure is not to scale. the continuum. The output power of the NB Ti:sapphire laser for the second transition is reduced to approximately $3 \mathrm{~mW}$ in order to minimize spectrum broadening due to saturation of resonances.

\section{LIST}

The LIST is coupled downstream of the target at a distance of $2.5 \mathrm{~mm}$ from the exit of the ion-source hot cavity as illustrated in Fig. 1. This distance is twice shorter than in the preceding on-line test at ISOLDE and has led to an improvement of the laser-ionization efficiency by a factor of 2.5 in this work [31].

Figure 3 shows a transverse cut through the LIST. The LIST consists of two circular electrodes (repeller and extractor electrodes) with central bore diameters of $11 \mathrm{~mm}$ at each end of a cylindrical housing of $90-\mathrm{mm}$ length and 38-mm diameter. The latter shields the enclosed radio-frequency quadrupole (RFQ) ion-guide structure from external fields such as the field of the ISOLDE extraction electrode (up to $60 \mathrm{kV}$ ) that is installed about $6 \mathrm{~cm}$ after the LIST in the ISOLDE beam line (see Fig. 1). The RFQ shield is electrically connected with the end electrode and the base of the target chamber, while the potential $U_{\text {rep }}$ of the front electrode (the so-called "repeller") could be controlled remotely in the range of $\pm 500 \mathrm{~V}$.

Ions inside the LIST RFQ structure are guided along the axis of the device toward the extractor of the ISOLDE mass separator by a weak longitudinal potential gradient between the repeller and LIST extractor electrode (see Fig. 3). The RFQ structure with quadrupole rods of $10-\mathrm{mm}$ diameter and a free-field radius of $7.5 \mathrm{~mm}$ provides transverse 


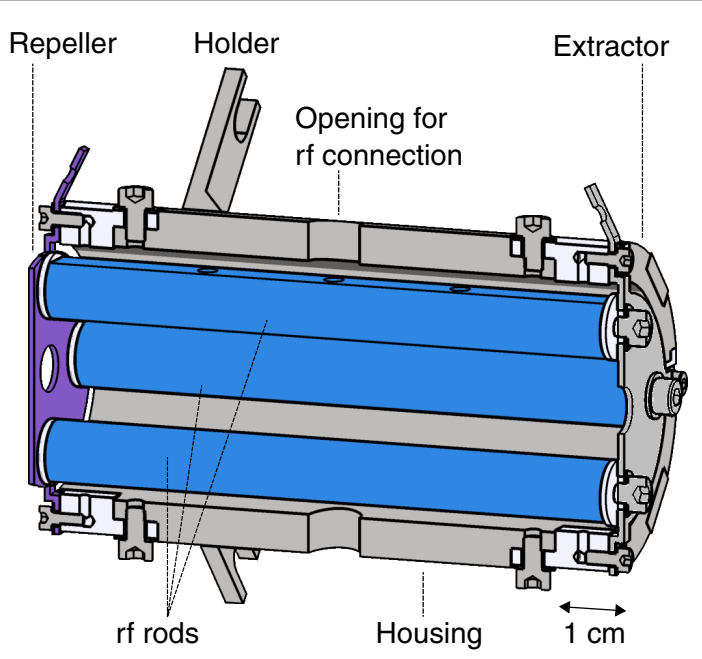

FIG. 3. A transverse cut through the LIST with its most important parts. Insulators are colored in light gray.

confinement of ions as they drift through the LIST toward the extraction field. It operates with a sinusoidal rf signal of frequency $f_{\text {rf }}=1.15 \mathrm{MHz}$ and an amplitude of up to $V_{\mathrm{rf}}=$ $500 \mathrm{~V}_{p p}$. While resonance laser ionization occurs wherever there is a laser-atom overlap (inside the hot cavity and along the axis of the LIST cavity), by adjusting the potential $U_{\text {rep }}$ to the repeller electrode, it is possible to select between two modes of operation.

(1) The first is the ion-guide mode (typically $U_{\text {rep }}=-50 \mathrm{~V}$ ): Ions from the hot cavity pass the repeller electrode and are guided by the RFQ to the extraction region. This mode of operation is the equivalent of the standard hot-cavity RILIS configuration.

(2) The second is the LIST mode (typically $U_{\text {rep }}=+7$ to $+50 \mathrm{~V}$, depending on the contaminant): Hotcavity ions are repelled, and only ions created inside the LIST structure are extracted. This mode is the high-selectivity mode, which provides a suppression of surface ions of at least $10^{3}$ ions/s but results in a laser-ionization efficiency drop by a factor of 20 , due to the relatively poor geometrical overlap of the laser and atom beams inside the RFQ.

For a more detailed description of the technical aspects of the LIST and a summary of the performance during this and earlier on-line tests, we refer to Refs. [31,36].

\section{The Windmill $\alpha$-decay detection setup}

The Windmill $\alpha$-decay detection setup [38] consists of a rotatable wheel, which holds ten thin carbon foils (10-mm diameter, $20 \mu \mathrm{g} / \mathrm{cm}^{2}$ [42]). The ion-beam implantation site is surrounded by two silicon detectors covering $51 \%$ of $4 \pi$ for the detection of charged particles [43]. One of the silicon detectors has an 8-mm aperture to allow the beam to pass through. The wheel can be rotated to remove longlived activity from the implantation position. A decay position is also equipped with two silicon detectors to observe the remaining decaying activity immediately after the first wheel motion. The data are collected with a triggerless acquisition system, which allows extraction of the time structure and appointment of coincidences between the detectors as well as with the laser pulses.

\section{PERFORMANCE OF THE LIST}

\section{A. Suppression of surface-ionized ions}

The suppression of surface-ionized ions is studied with beams of radioactive francium produced on-line at ISOLDE. The francium ions cover a wide range of masses, from $A=200$ [44] to 233 [45]. Their yields vary from $<1$ to $>10^{8}$ atoms per second, depending on the reaction cross section, the diffusion and effusion properties of the target, and the half-life of each isotope [46]. In the region of $A \geq 220$, the yields are $10^{7}$ ions per second [46], while the predicted yields of the isobaric polonium atoms are $<10^{3}$.

In order to demonstrate the suitability of the LIST in the region of the nuclear chart with $A \sim 220$, the suppression of ${ }^{218} \mathrm{Fr}$ has been studied. In Fig. 4, the difference in the $\alpha$-decay energy spectrum between the LIST data (blue lines) and standard surface ionization (red lines) is presented. The standard-surface-ion spectrum is acquired with the same detection setup as the LIST data but with a different target unit connected through a different separator magnet (HRS) with a higher mass-resolving power of $R \approx 7000$ but reduced transmission. The beam is purified in both cases using the pulsed-release technique [23], with beam-gate delays of $200 \mathrm{~ms}$ for the surface data and $100 \mathrm{~ms}$ for the LIST data, respectively. Considering the half-life of

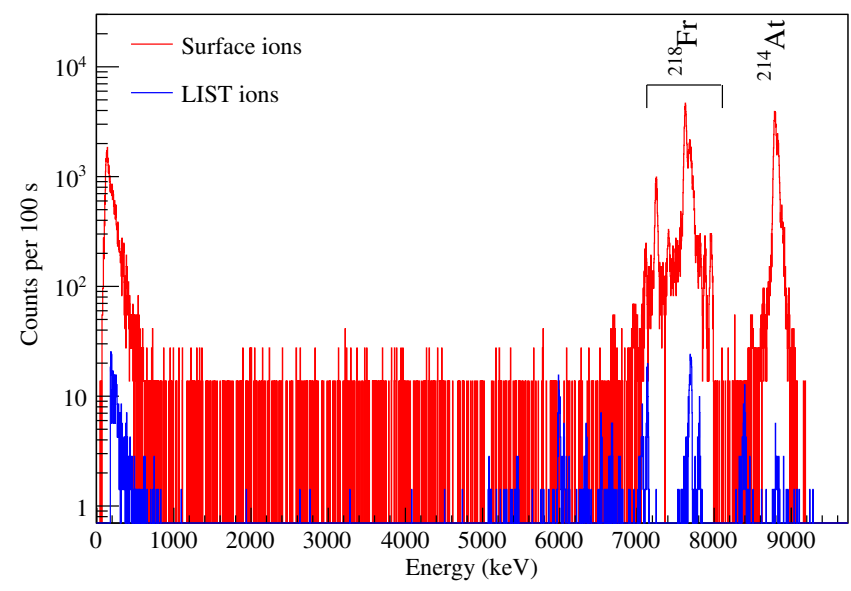

FIG. 4. Comparison of the $\alpha$-decay energy spectra for $A=218$ obtained in two different runs using the LIST to suppress the surface ions (blue lines) and using a standard-surface-ion source (red lines). The same measurement setup is used in both experiments. A beam-gate waiting time for the pulsed-release technique of $100 \mathrm{~ms}$ is applied for measurement with the LIST and $200 \mathrm{~ms}$ for the measurement with the surface-ion source. Both data sets are normalized to counts per $100 \mathrm{~s}$. 
${ }^{218} \operatorname{Fr}\left(T_{1 / 2}=22 \mathrm{~ms}\right.$ and $1 \mathrm{~ms}$ [47]), the suppression effect from the pulsed-release technique is expected to be greater for the surface data. In spite of the smaller transmission and the pulsed-release technique, the presence of ${ }^{218} \mathrm{Fr}$ in the beam is significant in the surface data, as demonstrated by its characteristic $\alpha$ decay and that of its daughter nucleus ${ }^{214} \mathrm{At}$. It can be explained by the decay of radiogenic ${ }^{222} \mathrm{Ac}$ $\left(T_{1 / 2}=63 \mathrm{~s}\right)$ inside the target, which results in the continuous production of ${ }^{218} \mathrm{Fr}$, independent of the proton impact on the target.

On the contrary, the LIST data show only trace amounts of ${ }^{218} \mathrm{Fr}$. The suppression is applied on the ion source rather than on the timing of the production mechanism. As a consequence, the suppression power of the LIST is independent of the irradiation pattern of the target and does not differentiate between the directly produced ions and those arising from the decay of a precursor accumulated in the target material.

The effect of the repeller in both ion-guide and RILIS modes has been studied in greater detail with beams of ${ }^{205,212} \mathrm{Fr}$. The ion-beam rate for each isotope as a function of the applied voltage is shown in Fig. 5. The ${ }^{205} \mathrm{Fr}$ data are collected with the Windmill while ${ }^{212} \mathrm{Fr}$ is studied with a Faraday cup. In the case of ${ }^{205} \mathrm{Fr}$, the highest suppression factor from the ion-guide mode to the LIST mode is 2540 . It is, however, only 70 for ${ }^{212} \mathrm{Fr}$.

In Fig. 5, it is observed that the intensities of ${ }^{205} \mathrm{Fr}$ and ${ }^{212} \mathrm{Fr}$ have a different dependency on the repeller potential. The intensity of the ${ }^{205} \mathrm{Fr}$ ions drops rapidly for positive repeller voltages. This rapid drop is due to most of the ions coming from the hot cavity being directly repelled by the repeller potential and the few ions that are observed for

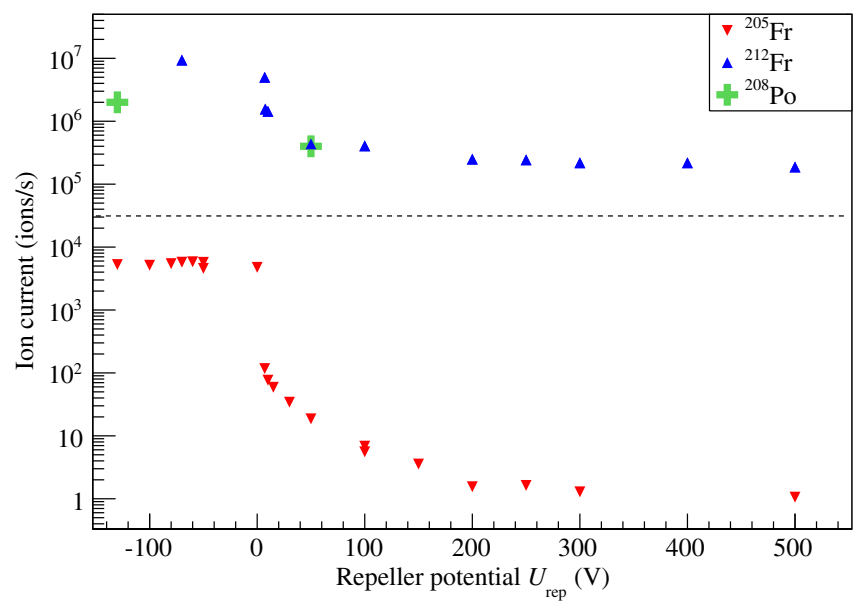

FIG. 5. Effect of the repeller potential on the ion current of laser-ionized polonium and surface-ionized francium isotopes. Negative voltages correspond to the ion-guide mode, while positive voltages correspond to the LIST mode. Data taken with different techniques are normalized to ions per second, and the detection limit for the Faraday-cup measurements is shown with the dotted line. positive repeller potentials being ions that are ionized closed to the repeller electrode by secondary ionization mechanisms (e.g., reionization of neutralized francium that condenses onto the repeller electrode). These ions have an offset of the kinetic energy corresponding to the potential at this position (approximately $0.6 \times U_{\text {rep }}$ on the longitudinal axis at the repeller position), which in turn leads to losses inside the separator magnet due to the limited energy acceptance. This dependency on the repeller potential is also observed for the laser-ionized ions, most of which are ionized close to the repeller electrode and whose intensity decreases with increased repeller potential $U_{\text {rep. This }}$ particular dependency is evidenced with the study of the ion time profile in Ref. [31]. For ${ }^{212} \mathrm{Fr}$, the intensity remains stable for repeller bias voltages from +50 to $+500 \mathrm{~V}$.

This lack of sensitivity to the bias voltages indicates that most of these ions are not ionized in the direct vicinity of the repeller electrode, whose penetration inside the LIST cavity is short but efficient. On the contrary, these ions are ionized alongside the longitudinal axis in the center of the LIST where the repeller potential and the potential of the ISOLDE extraction electrode do not penetrate, and only the initial thermal energy and the RFQ potential weak longitudinal potential gradient is guiding the ions. The significant difference in kinetic energies of laser-ionized ions and contaminants allows us to increase the selectivity in future experiments by tuning the magnetic field of the separator magnet, which has an energy acceptance of $50 \mathrm{eV}$.

\section{B. Alternative ionization mechanisms}

In order to better understand the difference in suppression of those isotopes, further studies of the ions' time profile are performed.

The time profile of the release of elements from the target material after the impact of a proton pulse is phenomenologically known to consist of a fast rise time and a long exponential decay [48]. In the case of alkali elements, the release is mostly contained within the 1.2-s separation between two proton pulses irradiating the target $[46,48]$. This release profile results in the francium beams to be structured with peaks synchronized with the proton-pulse impact on target.

The current of ${ }^{212} \mathrm{Fr}$ in ion-guide mode shows such a structure, but it disappears completely for a repeller voltage $>+7 \mathrm{~V}$. Although the suppression is only a factor of 70 , the observation of a continuous output indicates that the ions are not originating from the proton impact on target.

The alternative is that those ions are produced inside the LIST itself. Two parameters should be considered:

(1) the origin of the atoms and

(2) the ionization mechanism.

There are two possible origins for the atoms: evaporation of condensed atoms on the rods or decay products from condensed atoms. In the case of ${ }^{212} \mathrm{Fr}$, the signal observed in the Faraday cup in LIST mode is decaying with a 20-min 
half-life, corresponding to that of ${ }^{212} \mathrm{Fr}$, while its possible precursors $\left({ }^{212} \mathrm{Ra}\right.$ from $\beta$ decay and ${ }^{216} \mathrm{Ac}$ from $\alpha$ decay) have much shorter half-lives (13 s [49] and $44 \mathrm{~ms}$ [50], respectively). The long half-life behavior shows that it is a directly evaporated atom sample and not a decay product.

There are also several other possible ionization mechanisms: surface ionization off the rods, electron-impact ionization, and nonresonant laser ionization. The surface ionization is unlikely as the rods are shielded from the hot ion source and should not be sufficiently hot for surface ionization to occur. The signal is also not responding to the laser radiation. It is therefore believed to arise from electron impact following the acceleration of electrons by the rf field of the LIST quadrupoles and the field of the repeller electrode.

An example of decay-induced ion-beam production is also observed in beams of ${ }^{216} \mathrm{At}$ and ${ }^{217} \mathrm{Rn}$, as shown in the $\alpha$-decay energy spectrum of, e.g., $A=216$ in Fig. 6 . There are no precursors observed on the foil, meaning ${ }^{216} \mathrm{At}$ and ${ }^{217} \mathrm{Rn}$ are delivered as a beam. There is also no correlation observed with the proton impact on target. Finally, their half-lives (300 $\mu \mathrm{s}$ [50] and $540 \mu \mathrm{s}$ [51], respectively) are so short that those isotopes cannot be extracted from processes occurring inside the target, meaning that it comes directly from the LIST.

It is suggested that the parent nuclei, namely, ${ }^{220} \mathrm{Fr}$ and ${ }^{221} \mathrm{Ra}$, condensate on the rods of the rf structure of the LIST where they $\alpha$ decay, leaving their daughter nucleus in an ion form. While most of those ions recoil out of the pseudopotential produced by the RFQ, some are caught and extracted with the rest of the ions and delivered to the experiment.

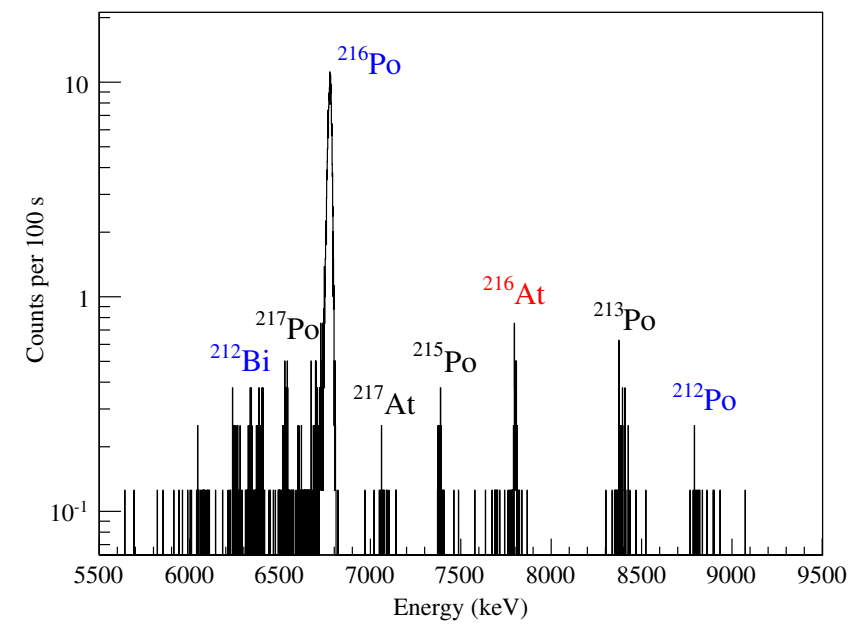

FIG. 6. $\alpha$-decay energy spectrum at $A=216$ using the LIST. A peak arising from the decay of ${ }^{216} \mathrm{At}\left(T_{1 / 2}=300 \mu \mathrm{s}\right.$ [50]) is highlighted in red. The peaks labeled in blue are part of the decay chain of ${ }^{216} \mathrm{Po}$, which is partially enhanced with $255-\mathrm{nm}$ laser light; those labeled in black are coming from contamination on the foils from earlier collections.
The production rates of ${ }^{216} \mathrm{At}$ and ${ }^{217} \mathrm{Rn}$ are 0.1 ions per second. These two isotopes represent the shortest-lived radioactive ion beams ever produced at ISOLDE since ${ }^{14} \mathrm{Be}$ $\left(T_{1 / 2}=4.35 \mathrm{~ms}\right)$ [52].

This additional source of nonresonant ions can be reduced in future designs of the LIST by producing an RFQ with thinner rods for a smaller surface area on which to condensate. Alternatively, this current design can be used to produce exotic beams otherwise not accessible at ISOL facilities.

\section{Laser ionization}

It has been observed in previous studies that the efficiency of the laser ionization in the LIST mode is lower than in the ion-guide mode $[31,36]$. The losses are mostly due to the reduced spatial overlap of the atomic flux and the laser beams inside the LIST cavity in contrast to the 3-mm-diameter hot cavity that is used in normal RILIS operation or in ion-guide mode. The atomic beam divergence in the LIST cavity leads to a fast reduction of the atom density alongside the longitudinal axis as a function of the distance to the hot cavity. The ionizing lasers provide beams of 3-mm diameter, and a large fraction of the atoms is not irradiated. The interest in the LIST relies on the grounds that losses are smaller for the ions of interest than the suppression is for the contaminants.

Similarly to the case of ${ }^{218} \mathrm{Fr}$, the rates of ionization of polonium in a standard laser ion-source configuration (RILIS) and with the LIST are compared in Fig. 7. ${ }^{196} \mathrm{Po}$ is laser ionized, and its $\alpha$-decay energy spectrum is recorded with the Windmill. The RILIS data are collected with a different configuration of the Windmill setup with

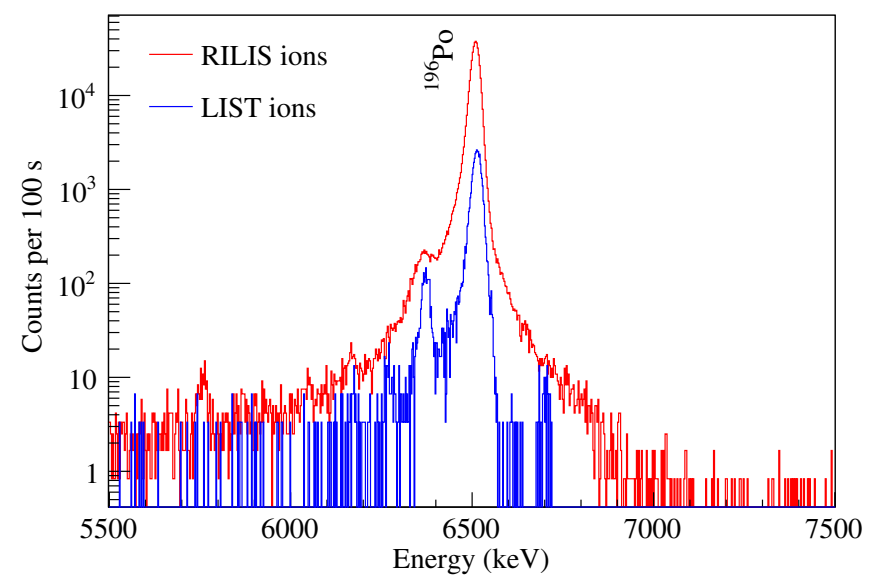

FIG. 7. Comparison of the $\alpha$-decay energy spectra obtained for $A=196$ with the lasers on polonium during the LIST run (blue lines) and with a standard RILIS ion source (red lines). The standard RILIS ions are studied with an earlier version of the experimental setup [40]. Both data sets are normalized to counts per $100 \mathrm{~s}$ and to the solid-angle coverage of the current version of the Windmill setup. 
smaller solid-angle coverage [40] and are corrected accordingly. Both data sets are normalized to counts per $100 \mathrm{~s}$. The RILIS laser system is also different for the two measurements, which can induce differences in the ionization efficiency. In both spectra, the presence of ${ }^{196} \mathrm{Po}$ is clearly visible, reduced by a factor of 20 with the LIST.

Alongside the study of francium suppression versus repeller potential, a reduction of laser-ionization efficiency by a factor of 5 is observed for ${ }^{208} \mathrm{Po}$ for the transition from ion-guide mode to LIST mode, as shown in Fig. 5. However, this number is also dependent on the proton current on target, relating to the total number of charged particles in the hot cavity, and on the size and space position of laser beams [36]. In other measurements, loss factors of 10 and 20 are measured for polonium and magnesium, respectively. In total, a net improvement of a factor $>500$ in beam purity (selectivity) can be reached in the cases where no alternative ionization mechanisms are present [31].

\section{Resonance line profile}

The linewidth and centroid of the 843.4-nm transition are systematically studied with ${ }^{208}$ Po. The resonance spectra are recorded using Faraday-cup detection subsequently in both LIST and ion-guide modes and are shown in Fig. 8. The spectrum of the same isotope studied in the same way for the standard RILIS with a different target unit is shown for comparison.

The full width at half maximum of the single-peak resonance profiles are very similar for all three modes of operations, ranging between 2.2 and $2.5 \mathrm{GHz}$. This profile is defined by the Doppler width associated with the velocity distribution of the atoms in the ion source (source

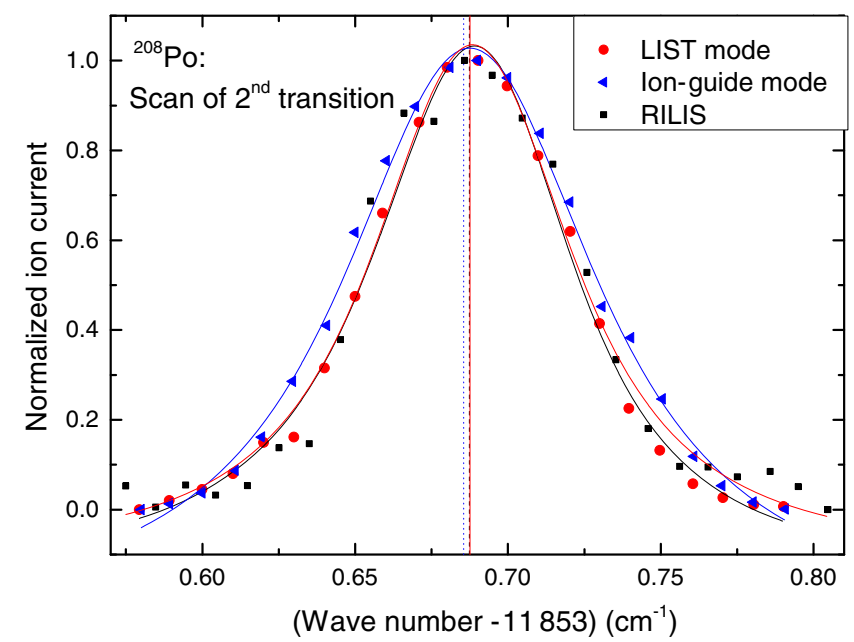

FIG. 8. Resonance line profile of the transition at $843.4 \mathrm{~nm}$ in ${ }^{208}$ Po recorded using Faraday-cup detection for both operation modes of the LIST (FWHM $\sim 2.5 \mathrm{GHz}$ ) and for a standard RILIS (FWHM $\sim 2.2 \mathrm{GHz}$ ). The lines along the data points represent the best fits to the data [38]. The vertical lines highlight the centroids extracted from these fits. temperature and atom-beam divergence) and by the spectral density of the laser power [38]. The small differences arise from drifts in the laser system over time. Those fit within the standard fluctuations of the operating parameters of the RILIS lasers and have no impact for the study of hyperfine structures and isotope shifts of radioactive isotopes.

The transition centroids are $11853.6855(30) \mathrm{cm}^{-1}$ in ion-guide mode, $11853.6875(30) \mathrm{cm}^{-1}$ in LIST mode, and $11853.6873(30) \mathrm{cm}^{-1}$ with the standard RILIS. All measurements agree within each other's uncertainty. This agreement highlights that the different conditions of laser-atom interactions in the studied operation modes of LIST and RILIS do not introduce any systematic shift in the resonance frequency and further confirms that the LIST is a suitable setup to perform in-source laser spectroscopy.

\section{LASER SPECTROSCOPY OF ${ }^{217}$ PO}

\section{A. Measurement}

Data acquisition is performed in the same manner as the previous polonium campaigns at ISOLDE [38], measuring the $\alpha$-decay rates at the Windmill detection setup while scanning the laser frequency of the 843.4-nm transition. A newly developed laser-scanning control [53] is synchronized to the PS Booster super cycle (46.8 s) to ensure the reproducibility of the target irradiation at each frequency step. The pulsed-release technique [23] with a beam-gate waiting time of $100 \mathrm{~ms}$ after proton impact is applied to partially suppress the tail of the mass distribution of the short-lived, neighboring isobar ${ }^{218} \mathrm{Fr}\left(T_{1 / 2}=22 \mathrm{~ms}\right)$.

Laser-frequency scans are recorded at mass $A=217$ and additionally at $A=218$ for the determination of the isotope shift. Because of the limited resolution of the GPS, ${ }^{216} \mathrm{Po}$ is observed simultaneously in the scans for mass $A=217$, rendering a specific reference scan for mass $A=216$ unnecessary. A further advantage of having both isotopes in the same mass spectra is that it allows for a direct measurement of the isotope shift between these two isotopes. In this approach, ${ }^{216} \mathrm{Po}$ serves as a reference isotope avoiding any laser- or acquisition-related frequency shifts $[54,55]$. For mass $A=219$, a scan is performed, but insufficient statistics prevent the determination of the hyperfine structure or isotope shift.

\section{B. $\alpha$-decay energy spectra}

Figure 9 shows representative $\alpha$-decay energy spectra measured by the silicon detector behind the carbon foil and the mass separator set to (a) $A=217$ and (b) $A=218$ and integrated over all frequency steps of the laser scan for ${ }^{216,217} \mathrm{Po}$ and ${ }^{218} \mathrm{Po}$, respectively.

In the case of mass $A=217$, the two strongest lines belong to laser-ionized ${ }^{217} \mathrm{Po}\left(E_{\alpha}=6536 \mathrm{keV}\right)$ and ${ }^{216} \mathrm{Po}$ $\left(E_{\alpha}=6778 \mathrm{keV}\right)$. The next significant peak is ${ }^{217} \mathrm{At}$ $\left(E_{\alpha}=7070 \mathrm{keV}\right)$, which is produced by $\beta^{-}$decay (5\% 

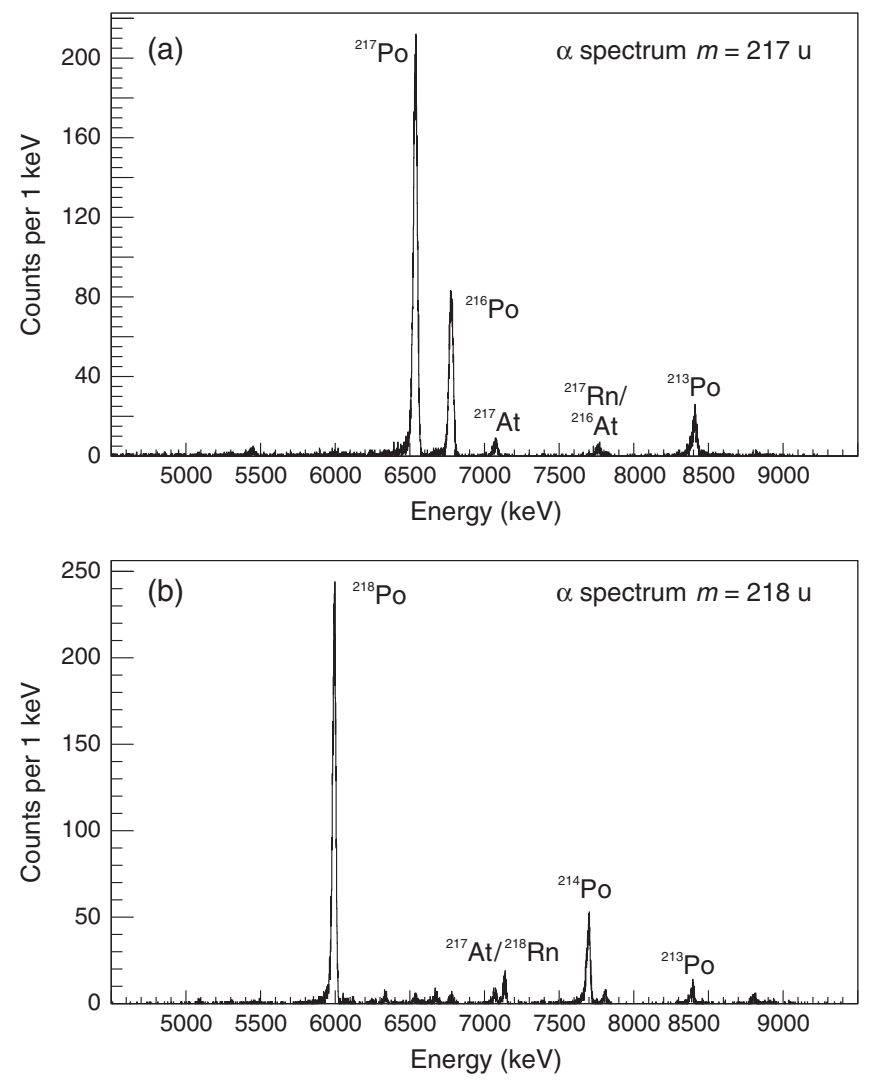

FIG. 9. $\alpha$-decay energy spectra for (a) $A=217$ and (b) $A=218$ obtained by the silicon detector behind the implantation point and integrated over all frequency steps of the laser scan for ${ }^{216,217} \mathrm{Po}$ and ${ }^{218} \mathrm{Po}$, respectively.

branch) from ${ }^{217} \mathrm{Po}$. Similarly, ${ }^{213} \mathrm{Po}\left(E_{\alpha}=8376 \mathrm{keV}\right)$ is produced from ${ }^{217} \mathrm{At} \stackrel{\alpha}{\rightarrow}{ }^{213} \mathrm{Bi} \stackrel{\beta}{\rightarrow}{ }^{213} \mathrm{Po}$ or ${ }^{217} \mathrm{Po} \stackrel{\alpha}{\rightarrow}{ }^{213} \mathrm{~Pb} \stackrel{\beta}{\rightarrow}$ ${ }^{213} \mathrm{Bi} \stackrel{\beta}{\rightarrow}{ }^{213} \mathrm{Po}$.

Traces of ${ }^{217} \mathrm{Rn}\left(E_{\alpha}=7741 \mathrm{keV}, T_{1 / 2}=540 \mu \mathrm{s}\right)$ and ${ }^{216} \mathrm{At}\left(E_{\alpha}=7802 \mathrm{keV}, T_{1 / 2}=300 \mu \mathrm{s}\right)$ are also visible in the spectrum, as discussed previously.

The $\alpha$-decay energy spectrum for mass $A=218$ is dominated by a well-isolated peak of ${ }^{218} \mathrm{Po} \quad\left(E_{\alpha}=\right.$ $6002 \mathrm{keV})$. The second-strongest peak arises from $\alpha$ decay of ${ }^{214} \mathrm{Po}\left(E_{\alpha}=7687 \mathrm{keV}\right)$, which is produced in the carbon foils in the decay chain of ${ }^{218} \mathrm{Po}$ : ${ }^{218} \mathrm{Po} \stackrel{\alpha}{\rightarrow}{ }^{214} \mathrm{~Pb} \stackrel{\beta}{\rightarrow}{ }^{214} \mathrm{Bi} \stackrel{\beta}{\rightarrow}{ }^{214} \mathrm{Po} . \quad{ }^{213} \mathrm{Po} \quad\left(E_{\alpha}=8376 \mathrm{keV}\right)$ appears due to contamination of the carbon foils by decay products of ${ }^{217}$ Po during preceding laser scans at $A=217$, as described previously. Within these decay chains, ${ }^{213} \mathrm{Bi}$ serves as a waiting point due to its relatively long half-life of 45.59 min. The weaker peaks, ${ }^{217}$ At $\left(E_{\alpha}=7067 \mathrm{keV}\right)$ and ${ }^{218} \mathrm{Rn}\left(E_{\alpha}=7129 \mathrm{keV}\right)$, are most likely produced via nonlaser-induced ionization inside the LIST cavity. Additionally, traces of ${ }^{216} \mathrm{Po}\left(E_{\alpha}=6778 \mathrm{keV}\right),{ }^{218} \mathrm{At}\left(E_{\alpha}=6693 \mathrm{keV}\right)$, and ${ }^{217} \mathrm{Po}\left(E_{\alpha}=6536 \mathrm{keV}\right)$ are observed between ${ }^{217} \mathrm{Po}$ and ${ }^{217}$ At.
Apart from the decay products of the polonium isotopes and the rather weak peaks of ${ }^{216} \mathrm{At}$ and ${ }^{217} \mathrm{Rn}$, no additional lines are visible and the polonium peaks are well isolated. A significant consequence of the LIST application is the absence of the ${ }^{213,220,221} \mathrm{Fr} \alpha$-decay lines in the $\alpha$-decay energy spectra at $A=217$ and $A=218$, in contrast to the previous experiments $[16,38]$, demonstrating the effective suppression of surface-ionized isotopes by the LIST.

\section{Laser resonance spectra}

Laser spectra for ${ }^{216-218}$ Po (see Fig. 10) are obtained by integrating the counts in the respective $\alpha$-decay lines seen in Fig. 9 for every laser-frequency step. The even-even isotopes ${ }^{216,218}$ Po show the single resonance for the groundstate spin $I^{\pi}=0^{+}$and a clear isotope shift with respect to each other, while the odd- $A$ isotope ${ }^{217}$ Po scan exhibits a complex hyperfine structure.

The spectra are fitted using the formalism described in Ref. [38]. The isotope shift $\delta \nu$ and the hyperfine parameters $A$ and $B\left({ }^{217}\right.$ Po only) are extracted. They are presented in Tables I and II. Considering the uncertainty of the groundstate spin of ${ }^{217} \mathrm{Po}[56,57]$, the hyperfine structure is fitted with $I=9 / 2$ and $11 / 2$ and both results are presented in the tables. No real difference in the fit is found between the different assignments.

\section{Changes in the mean-square charge radii}

The changes in the mean-square charge radii $\delta\left\langle r^{2}\right\rangle$ are extracted from the $\delta \nu$ following the formalism presented in

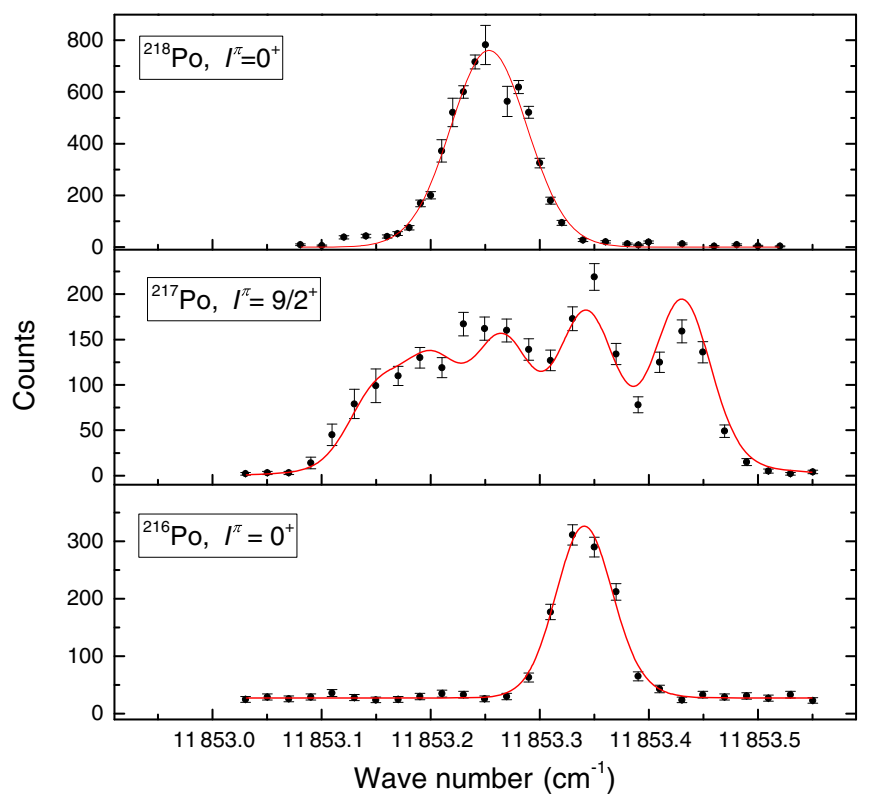

FIG. 10. Laser resonance spectra of the neutron-rich $216,217,218$ Po isotopes recorded in the LIST mode. The red curves are the fits to the data according to the nuclear spin given on each figure. 
TABLE I. Isotope shifts $\delta \nu_{A, 216}$ and changes in the mean-square charge radii $\delta\left\langle r^{2}\right\rangle$ of ${ }^{216,217,218}$ Po. The results for ${ }^{216,218}$ Po from previous RILIS measurements [16] are shown for comparison and to calculate $\delta \nu_{A, 210}$. Statistical uncertainties are given in parentheses, and systematic uncertainties originating from the atomic parameters are given in the curly brackets. For ${ }^{217}$ Po, the results for fitting with both $I=9 / 2$ and $11 / 2$ are presented, showing no significant difference.

\begin{tabular}{|c|c|c|c|c|c|c|c|}
\hline \multirow[b]{2}{*}{ Isotope } & \multirow[b]{2}{*}{$I^{\pi}$} & \multirow[b]{2}{*}{$T_{1 / 2}(\mathrm{~s})$} & \multicolumn{2}{|c|}{ Previous work (RILIS) [16] } & \multicolumn{3}{|c|}{ This work (LIST) } \\
\hline & & & $\delta \nu_{A, 210}(\mathrm{GHz})$ & $\delta\left\langle r^{2}\right\rangle_{A, 210}\left(\mathrm{fm}^{2}\right)$ & $\delta \nu_{A, 216}(\mathrm{GHz})$ & $\delta \nu_{A, 210}(\mathrm{GHz})$ & $\delta\left\langle r^{2}\right\rangle_{A, 210}\left(\mathrm{fm}^{2}\right)$ \\
\hline${ }^{216} \mathrm{Po}$ & $0^{+}$ & 0.145 & $-8.820(110)$ & $0.733(10)\{5\}$ & $\ldots$ & $\ldots$ & $\ldots$ \\
\hline${ }^{217} \mathrm{Po}$ & $\left(9 / 2^{+}\right)$ & 1.46 & $\ldots$ & $\ldots$ & $1.060(150)$ & $-9.880(200)$ & $0.821(17)\{6\}$ \\
\hline & $\left(11 / 2^{+}\right)$ & & $\ldots$ & $\ldots$ & $1.165(150)$ & $-9.985(200)$ & $0.830(17)\{6\}$ \\
\hline${ }^{218} \mathrm{Po}$ & $0^{+}$ & 186 & $-11.524(125)$ & $0.958(10)\{7\}$ & $2.586(100)$ & $-11.406(150)$ & $0.948(10)\{7\}$ \\
\hline
\end{tabular}

TABLE II. Hyperfine parameters, electromagnetic moments, and $g$ factors of ${ }^{207,211,217}$ Po from Ref. [38] (and references therein) and this work, respectively. Statistical uncertainties are given in parentheses, and the total uncertainties including systematic uncertainties originating from the reference isotope ${ }^{207} \mathrm{Po}$ are given in the curly brackets. For ${ }^{217} \mathrm{Po}$, the results for fitting with both $I=9 / 2$ and $11 / 2$ are presented.

\begin{tabular}{lccccrr}
\hline \hline Isotope & $I^{\pi}$ & $A(\mathrm{GHz})$ & $B(\mathrm{GHz})$ & $\mu\left(\mu_{N}\right)$ & $g$ & $Q_{S}(b)$ \\
\hline${ }^{207} \mathrm{Po}$ & $5 / 2^{+}$ & $0.564(1)$ & $0.367(7)$ & $0.793(55)$ & $0.317(22)$ & $0.28(3)$ \\
${ }^{211} \mathrm{Po}$ & $9 / 2^{+}$ & $-0.473(12)$ & $-1.2(1)$ & $-1.197(30)\{85\}$ & $-0.266(19)$ & $-0.77(8)\{15\}$ \\
${ }^{217} \mathrm{Po}$ & $\left(9 / 2^{+}\right)$ & $-0.437(20)$ & $+0.1(4)$ & $-1.106(50)\{90\}$ & $-0.246(20)$ & $+0.06(30)\{32\}$ \\
& $\left(11 / 2^{+}\right)$ & $-0.364(20)$ & $0.0(4)$ & $-1.126(50)\{90\}$ & $-0.205(16)$ & $0.00(30)\{32\}$ \\
\hline \hline
\end{tabular}

Refs. [16,58] using atomic parameters from large-scale atomic calculations [59]. The extracted $\delta\left\langle r^{2}\right\rangle$ are listed in Table I and are shown in Fig. 11.

The isotope shift $\delta \nu_{216,218}$ has been evaluated with both the standard RILIS configuration [16] and with the LIST. ${ }^{216} \mathrm{Po}$ is used as the reference isotope throughout this work, providing an ideal comparison value between the two

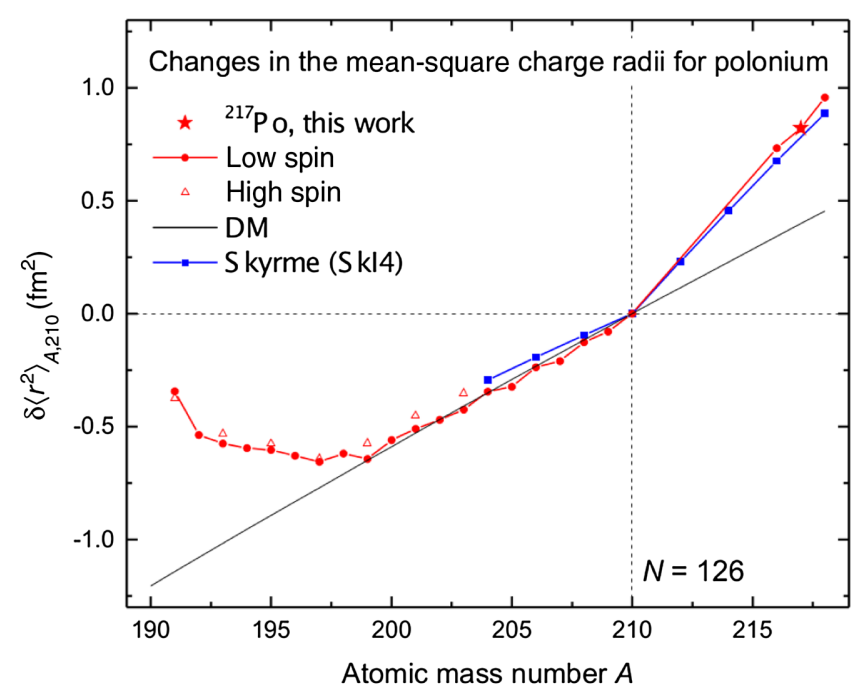

FIG. 11. Changes in the mean-square charge radii $\delta\left\langle r^{2}\right\rangle$ along the polonium isotope chain. The experimental data are shown in red $[16,58,60]$, the prediction from the spherical droplet model (DM) in black [61,62], and mean-field calculations in blue [63]. experimental setups. As given in Table I, $\delta \nu_{216,218}$ agrees within uncertainties of $1 \%$, removing the concern that the LIST might introduce systematic effects and validating its use for isotope-shift studies.

An outstanding feature of the $\delta\left\langle r^{2}\right\rangle$ in the region of interest is the odd-even staggering reversal already identified in ${ }_{86} \mathrm{Rn} \mathrm{[64],}{ }_{87} \mathrm{Fr}$ [37,65], and ${ }_{88} \mathrm{Ra}$ [66]. This phenomenon has been found to be correlated to the island of octupole deformation $[65,66]$, but the nature of the connection between the two phenomena remains under question [67]. The visibility of the odd-even staggering is enhanced by removing the even- $A$ trend from the $\delta\left\langle r^{2}\right\rangle$ by calculating the relative odd-even staggering parameter $\delta$ :

$\delta= \begin{cases}0 & \text { if } A \text { is even } \\ \delta\left\langle r^{2}\right\rangle_{A}-\frac{1}{2}\left(\delta\left\langle r^{2}\right\rangle_{A-1}+\delta\left\langle r^{2}\right\rangle_{A+1}\right) & \text { if } A \text { is odd. }\end{cases}$

This parameter $\delta$ is shown for the isotopes of even- $Z$ elements in Fig. 12. Odd- $A$ isotopes with $N \leq 126$ systematically show $\delta \leq 0$, as is characteristic across the nuclear chart. However, for $N>126$, odd-even staggering reversal is found for ${ }_{86} \mathrm{Rn}$ and ${ }_{88} \mathrm{Ra}$, with $\delta(N=133$, $135,137)>0$. This phenomenon is not observed in ${ }^{217} \mathrm{Po}_{133}$, which staggers in the usual negative way. The absence of staggering indicates that ${ }^{217} \mathrm{Po}$ is located outside the region of odd-even staggering reversal. More complex parameters exist to evaluate the odd-even 


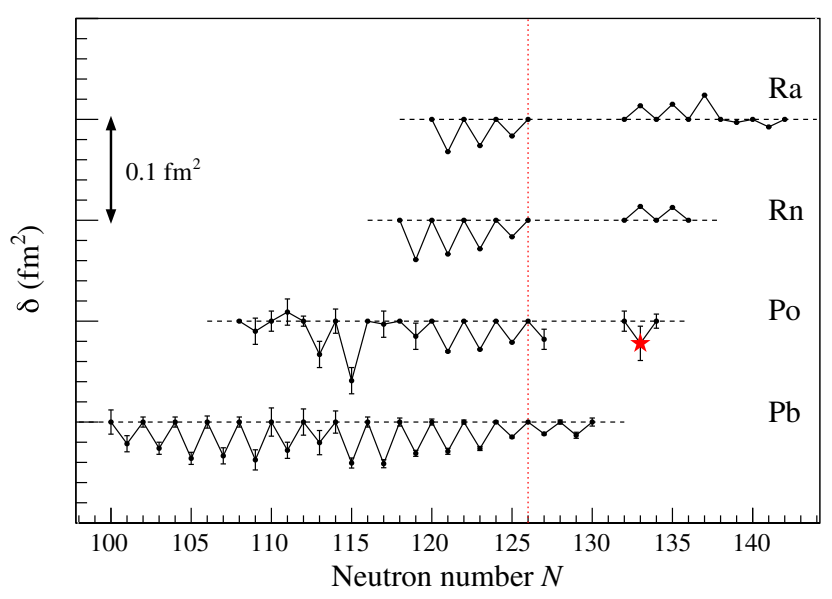

FIG. 12. Relative odd-even staggering $\delta$ in the $\delta\left\langle r^{2}\right\rangle$ of ${ }_{82} \mathrm{~Pb}$ [14,70-72], ${ }_{84} \mathrm{Po}[16,58,60],{ }_{86} \mathrm{Rn}[64,73]$, and ${ }_{88} \mathrm{Ra}[66,73]$ [see Eq. (1)]. Error bars from the original $\delta\left\langle r^{2}\right\rangle$ are shown for each point. The vertical offset between each chain is artificial for better display. The vertical line at $N=126$ marks the neutron-shell closure. For ${ }^{217} \mathrm{Po}_{133}$, only the solution for $I=9 / 2$ is shown, highlighted by the red star.

staggering, as discussed, e.g., in Refs. [68,69], but the polonium data at hand are too limited for a conclusive interpretation.

\section{E. Electromagnetic moments}

The electromagnetic moments $\mu$ and $Q_{S}$ are extracted following the formalism presented in Ref. [38] using ${ }^{207} \mathrm{Po}$ as a reference isotope. Resulting values for the final electromagnetic moments are given in Table II, together with the $g$ factors.

The latter data enable a comparison of different isotopes with similar nuclear configurations but different spins. The $g$ factors associated with either the quenched free-particle $g$ factor $g_{S}=0.6 g_{S, \text { free }}$ [74] or the single-particle $g$ factor [75] for a neutron occupying the $g_{9 / 2}$ orbital are, respectively, -0.255 and -0.294 with $I=9 / 2$. The measured data for the two isotopes ${ }^{211} \mathrm{Po}$ and ${ }^{217} \mathrm{Po}$, with respective $g$ factors of $-0.266(19)$ and $-0.246(20)$, show that in this respect, the two isotopes are very similar, both exhibiting the singleparticle value for $\nu g_{9 / 2}$.

It is worth mentioning that the $g$ factor for a single neutron occupying the $i_{11 / 2}$ orbital would be +0.177 [74] or +0.126 [75], both of which are inconsistent with the extracted value of -0.205 (16) for $I=11 / 2$. This discrepancy in the case of $I=11 / 2$ further supports the $I^{\pi}=$ $9 / 2^{+}$assignment, which is connected with the absence of octupole deformation [57], while the possibility of octupole deformation in the ground state of ${ }^{217} \mathrm{Po}$ is associated with the occupancy of the $i_{11 / 2}$ orbital [56].

The electric quadrupole moment for ${ }^{217} \mathrm{Po}$ is found to be consistent with 0 . It further supports the claim that this isotope does not display any static deformation in its ground state [57].

\section{DECAY SPECTROSCOPY OF ${ }^{219}$ PO}

\section{A. Measurement}

The acquisition cycles for ${ }^{219} \mathrm{Po}$ are determined by two parameters: the implantation period, during which the ion beam is delivered to the foil at the implantation position, and the possibly longer observation period, during which the decay is observed. Once a full cycle is completed, the activity at the implantation site is removed by turning the wheel, bringing the activated sample to the decay position and a fresh foil to the implantation position. The pulsedrelease technique [23] with a beam-gate waiting time of $100 \mathrm{~ms}$ after proton impact is applied to minimize isobaric

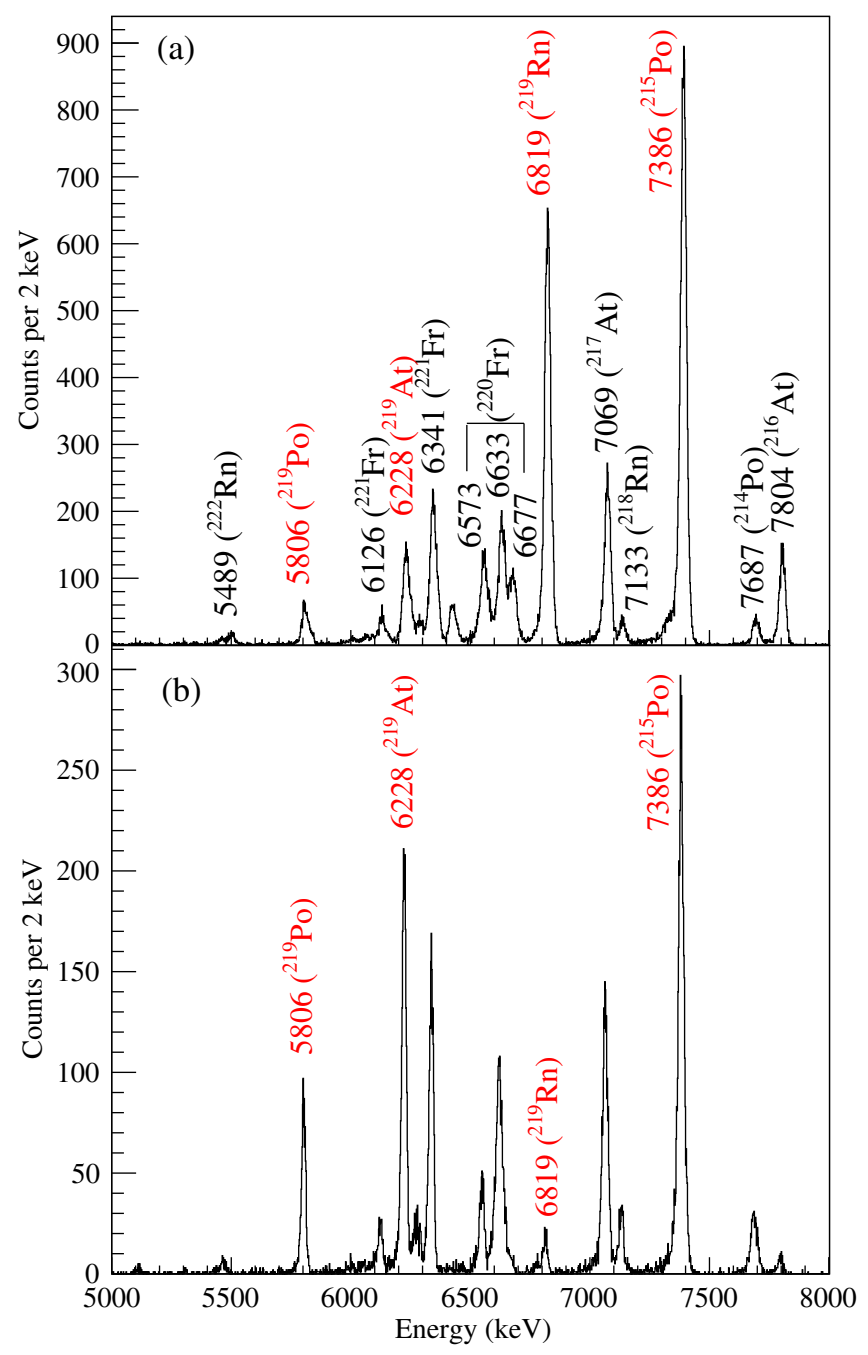

FIG. 13. $\alpha$-decay energy spectrum for $A=219$ at the Windmill (a) implantation and (b) decay positions using 300-s implantation and observation periods with the lasers on polonium. The $\alpha$-decay energies are in $\mathrm{keV}$. Decays originating from the implantation of ${ }^{219}$ Po are shown in red. 


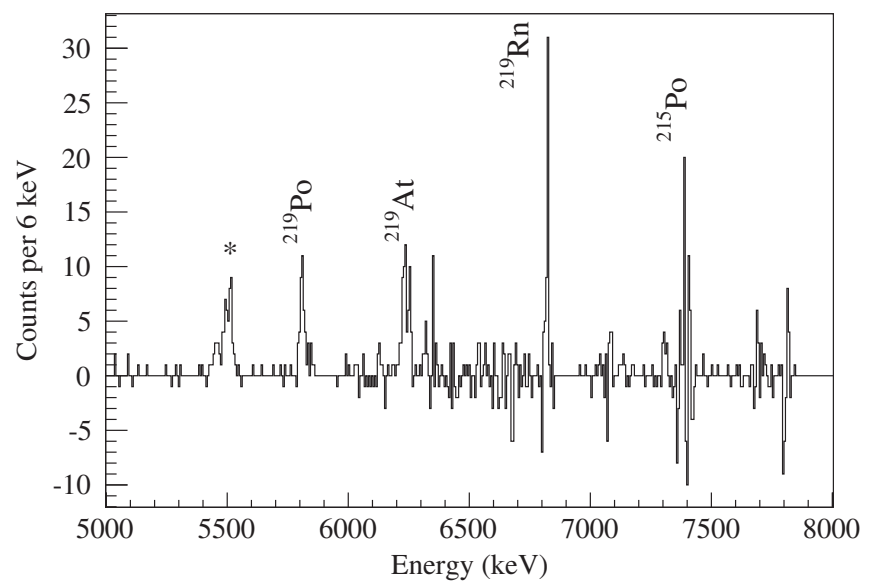

FIG. 14. Difference between the $\alpha$-decay energy spectrum for mass $A=219$ at the implantation position with lasers tuned to a resonance of polonium and lasers off, using 100-s implantation and 180-s observation times. The peak marked with an asterisk is ${ }^{222} \mathrm{Rn}$, a long-lived contaminant implanted on the laser-on carbon foil (see the text for details). The subtraction of two large peaks for ${ }^{219} \mathrm{Rn}$ and ${ }^{215} \mathrm{Po}$, arising from the direct production of ${ }^{219} \mathrm{Rn}$, results in high fluctuations in the counts around 6819 and $7386 \mathrm{keV}$.

${ }^{219} \mathrm{Fr}\left(T_{1 / 2}=21 \mathrm{~ms}\right)$ in addition to purification with the LIST.

\section{B. $\alpha$-decay spectra}

The $\alpha$-decay energy spectrum at $A=219$ is shown in Fig. 13 for the (a) implantation and (b) decay sites using 300 -s implantation and observation periods. Known $\alpha$ decay transitions from ${ }^{219} \mathrm{At},{ }^{219} \mathrm{Rn}$, and its daughter ${ }^{215} \mathrm{Po}$ from $\alpha$ decay are identified. The reduction in the intensity ratio of the $6819-\mathrm{keV}$ peak from the decay of ${ }^{219} \mathrm{Rn}$ with respect to ${ }^{219}$ At between the implantation and decay positions shows that most of the ${ }^{219} \mathrm{Rn}$ stems directly from the ion beam, rather than from in-foil $\beta$ decay of ${ }^{219}$ At. Since ${ }^{219} \mathrm{Rn}$ has a short half-life $\left(T_{1 / 2}=3.96 \mathrm{~s}\right)$ with respect to the observation cycle $(\Delta T=300 \mathrm{~s}), 99.7 \%$ of the implanted ${ }^{219} \mathrm{Rn}$ decays within the first $12 \mathrm{~s}$ of the observation at the decay position, while only small amounts are steadily produced via the $\beta$ decay of ${ }^{219} \mathrm{At}$ $\left(T_{1 / 2}=56 \mathrm{~s}\right)$. The intensity of the peak at $7386 \mathrm{keV}$, stemming from the $\alpha$ decay of ${ }^{215}$ Po, depends on both the $\alpha$ decay of ${ }^{219} \mathrm{Rn}$ and the $\beta$ decay of ${ }^{215} \mathrm{Bi}$, with a longer half-life $\left(T_{1 / 2}=7.7 \mathrm{~min}\right)$. Decays from neighboring masses are also seen: ${ }^{218} \mathrm{Rn}$, its daughter ${ }^{214} \mathrm{Po},{ }^{220,221} \mathrm{Fr}$, and their daughters ${ }^{216,217}$ At. The presence of directly ionized radon and francium originates from the processes described in the section on alternative ionization mechanisms. Finally, long-lived ${ }^{222} \mathrm{Rn}\left(T_{1 / 2}=3.825\right.$ days $)$ is also seen in the spectrum at $5489 \mathrm{keV}$, arising from one of the carbon foil, which has been contaminated in the course of the experimental campaign.

By comparing the spectra with and without laser irradiation, it is possible to identify the $\alpha$-decay lines related to polonium; see Fig. 14. The peaks observed in the lasers-off-subtracted spectrum are identified as arising from the $\alpha$ decay of ${ }^{219}$ Po and its progeny ${ }^{219} \mathrm{At},{ }^{219} \mathrm{Rn}$, and ${ }^{215} \mathrm{Po}$. As ${ }^{219} \mathrm{Rn}$ is also produced directly, the subtraction of two large peaks occurring in both spectra results in large fluctuations of the counts around 6819 and $7386 \mathrm{keV}\left({ }^{215} \mathrm{Po}\right)$.

The properties of those four isotopes are summarized in Table III. The $\alpha$-decay energies for ${ }^{219} \mathrm{Po}$ and ${ }^{219}$ At are determined to be $5806(5)$ and $6228(5) \mathrm{keV}$, respectively.

\section{Branching ratios}

Using collections made with $300 \mathrm{~s}$ for both implantation and observation periods, it is possible to deduce the branching ratios $b_{\alpha}$ and $b_{\beta}$ of $\alpha$ and $\beta$ decays, respectively, in the case of ${ }^{219} \mathrm{Po}$ and ${ }^{219}$ At. As seen in Fig. 15, the branching ratios of these isotopes can be determined by comparing the number of direct $\alpha$ decays with the number of $\alpha$ decays from their daughter nuclei via $\beta$ decay.

For ${ }^{219} \mathrm{At}$, the numbers of events at $6228 \mathrm{keV}$ ( $\alpha$ decay) and $6819 \mathrm{keV}$ ( $\beta$ decay) are compared with each other, including the known $b_{\alpha}\left({ }^{219} \mathrm{Rn}, 6819 \mathrm{keV}\right)=79.4(10) \%$ [76]. Only data from the decay position are considered, so that the directly implanted ${ }^{219} \mathrm{Rn}$ ions can be minimized and their influence disregarded. Recoils out of the carbon foil following $\beta$ decay are negligible. A branching ratio of $b_{\alpha}\left({ }^{219} \mathrm{At}\right)=93.6(10) \%$ is found. It is in reasonable agreement with the previous estimate of approximately 97\% [78] that was determined in the study of the $\alpha$ decay of natural ${ }^{227}$ Ac samples.

The same procedure is applied to ${ }^{219}$ Po by comparing the number of events at the implantation position at $5806 \mathrm{keV}$

TABLE III. Decay properties of ${ }^{219} \mathrm{Po}$ and its progenies ${ }^{219} \mathrm{At},{ }^{219} \mathrm{Rn}$, and ${ }^{215} \mathrm{Po}$.

\begin{tabular}{lcccccc}
\hline \hline Isotope & Half-life $(\mathrm{s})$ & $E_{\alpha}(\mathrm{keV})$ & $b_{\alpha}(\%)$ & Partial decay width $(\mathrm{keV})$ & Hindrance factor & Reference \\
\hline${ }^{219} \mathrm{Po}$ & $620(59)$ & $5806(5)$ & $28.2(20)$ & $75(10)$ & 1.5 & This work \\
${ }^{219} \mathrm{At}$ & $56(3)$ & $6228(5)$ & $93.6(10)$ & $107(8)$ & 1.1 & This work \\
${ }^{219} \mathrm{Rn}$ & $3.96(1)$ & $6819.1(3)$ & $79.4(10)$ & $6.1(1)$ & 11.4 \\
${ }^{215} \mathrm{Po}$ & $0.001781(4)$ & $7386.1(8)$ & $99.92(2)$ & $82(1)$ & {$[76]$} \\
\hline \hline
\end{tabular}

${ }^{\mathrm{a}}$ Half-life taken from Ref. [76]. 


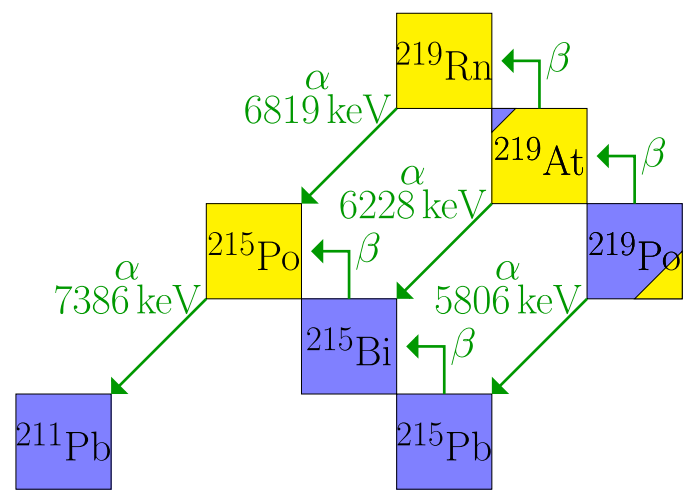

FIG. 15. Decay chain starting from ${ }^{219} \mathrm{Po}$ down to ${ }^{211} \mathrm{~Pb} . \alpha$ decaying isotopes are shown in yellow, $\beta$-decaying isotopes in blue, and possible decay paths are highlighted with an arrow.

( $\alpha$ decay) and $6228 \mathrm{keV}$ ( $\beta$ decay), taking into account the new branching ratios that have been determined for the decay of ${ }^{219}$ At. Since no ${ }^{219}$ At is directly implanted on the foils, the full data may be utilized. Once again, the $\beta$-decay recoils are ignored. A branching ratio of $b_{\alpha}\left({ }^{219} \mathrm{Po}\right)=$ $28.2(20) \%$ is found. The results are summarized in Table III.

\section{Half-life}

In the course of the experiment, a 1200-s decay-only period is recorded at the decay site. The half-life of ${ }^{219} \mathrm{Po}$ is determined to be 620(59) $\mathrm{s}$ by fitting the time behavior of the $\alpha$-decay peak at $5806 \mathrm{keV}$, shown in Fig. 16, to a single exponential decay curve.

\section{E. Hindrance factors}

The $\alpha$-decay hindrance factors (HFs) are calculated following the formalism of Rasmussen [79] by comparing to the $\alpha$ decay of ${ }^{218}$ Po [partial decay width $117(1) \mathrm{keV}$ ]. Values of $\mathrm{HF}\left({ }^{219} \mathrm{Po}\right)=1.5$ and $\mathrm{HF}\left({ }^{219} \mathrm{At}\right)=1.1$ are determined. Both isotopes exhibit, therefore, unhindered $\alpha$ decay,

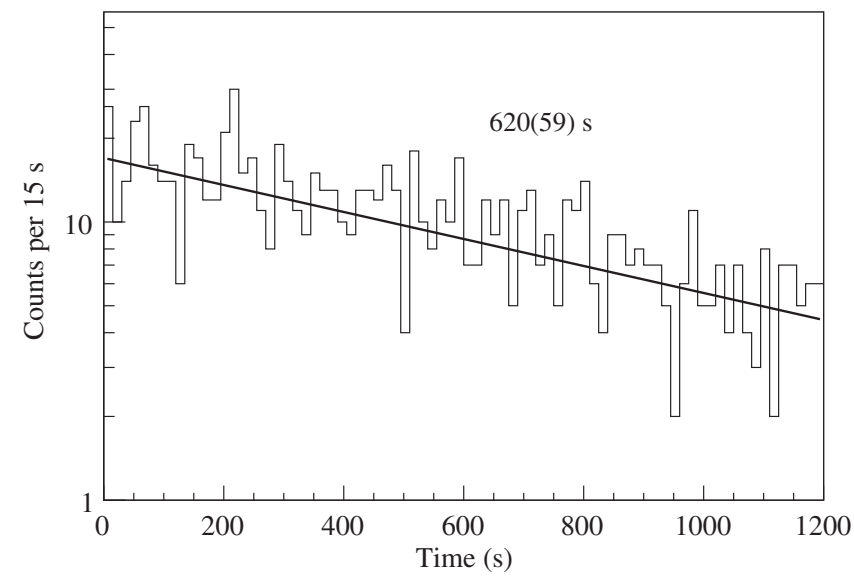

FIG. 16. Time behavior of the 5806-keV $\alpha$ decays of ${ }^{219} \mathrm{Po}$ during a 1200-s decay-only period of an implanted sample.

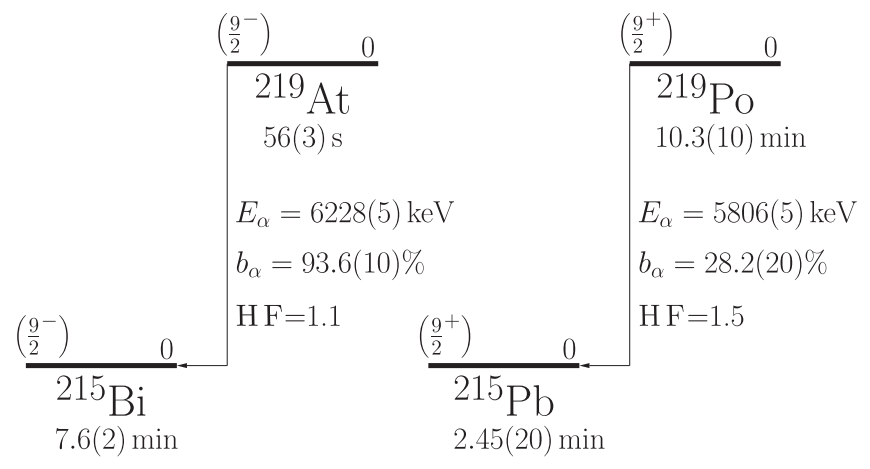

FIG. 17. $\alpha$ decay of ${ }^{219}$ At (left) and ${ }^{219}$ Po (right) with the information extracted from this work (see Table III) and Ref. [76].

which indicates that their nuclear ground-state spin and configuration are similar to their daughter nuclei ${ }^{215} \mathrm{~Pb}$ and ${ }^{215} \mathrm{Bi}$, respectively. In the case of ${ }^{219} \mathrm{Po}$, this configuration would correspond to a spin $I^{\pi}=\left(9 / 2^{+}\right)$arising from a valence neutron in the $\nu 2 g_{9 / 2}$ orbital [12]. This configuration is consistent with the predictions of the spherical shell model of the nucleus and suggests that ${ }^{219} \mathrm{Po}$ does not display any sign of octupole deformation. In the case of ${ }^{219} \mathrm{At}$, the spin is $I^{\pi}=\left(9 / 2^{-}\right)$, arising from a valence proton in the $\pi 1 h_{9 / 2}$ orbital. The complete information on the decay of ${ }^{219} \mathrm{Po}$ and ${ }^{219}$ At is shown in Fig. 17.

\section{CONCLUSIONS}

A substantial improvement of the purity of polonium ion beams produced with RILIS has been obtained due to implementation of the LIST. Experiments on in-source laser spectroscopy of ${ }^{216-218} \mathrm{Po}$ and nuclear decay study of ${ }^{219}$ Po have been performed using the LIST. This study represents the first use of the device for a dedicated experiment at CERN ISOLDE and its first use for on-line hyperfine structure and decay studies of radioisotopes. The comparison of the laser spectroscopy data with previously measured ${ }^{216,218}$ Po shows that no systematic effects were added by the use of the LIST. Furthermore, the enhanced selectivity of the device has enabled the study of isotopes with $A>218$ for the first time. This achievement opens up a new series of experiments in this region of the nuclear chart, with further experiments already planned on neutronrich ${ }_{81} \mathrm{Tl}$ and ${ }_{84} \mathrm{Po}$.

The systematic study of the $\delta\left\langle r^{2}\right\rangle$ odd-even staggering and of the nuclear electromagnetic moments indicates that ${ }^{217} \mathrm{Po}$ is located outside the region of reflection asymmetry, which would be associated with the region of odd-even staggering reversal. The study of more neutron-rich isotopes of polonium and of the neutron-rich ${ }_{85}$ At isotopes is, however, required to fully delineate those regions of interest. The combination of the LIST for laser spectroscopy with the ISOLTRAP multireflection time-of-flight mass spectrometer for efficient identification of beam 
composition and ion counting $[39,80]$ will render these investigations possible.

Finally, the nuclear decay properties of ${ }^{219}$ Po have been determined for the first time while knowledge on those for ${ }^{219} \mathrm{At}$ has been improved. The properties of ${ }^{219} \mathrm{Po}$ are consistent with the systematics of that region of the nuclear chart, for which the structure of the odd-mass polonium isotopes is dominated by a spherical neutron $g_{9 / 2}$ orbital.

\section{ACKNOWLEDGMENTS}

We acknowledge the support of the ISOLDE Collaboration and technical teams for providing excellent beams, the workshop of the Physics Institute of the Johannes Gutenberg-Universität Mainz for constructing the LIST, the ISOLDE Target and Ion Source Development team for their support in the development and operation of the LIST, and the GSI Target Laboratory for manufacturing the carbon foils. This work was supported by the Bundesministerium für Bildung und Forschung (BMBF, Germany) within the WolfgangGentner programme as well as through the consecutive project fundings of $06 \mathrm{Mz} 9181 \mathrm{I}, 06 \mathrm{Mz} 7177 \mathrm{D}$, and 05P12UMCIA, by FWO-Vlaanderen (Belgium), by GOA/2010/010 (BOF-KULeuven), by the IUAP-Belgian State Belgian Science Policy (BRIX network P7/12), by the U.K. Science and Technology Facilities Council (STFC), by the European Union within FP7 (ENSAR No. 262010), by the Slovak Research and Development Agency (Contract No. APVV-0105-10), by the Slovak grant agency VEGA, and the Reimei Foundation of JAEA (Contract No. 1/0576/13). T. E. C. was supported by STFC Ernest Rutherford Grant No. ST/J004189/1.

[1] A. Gottardo et al., New Isomers in the Full Seniority Scheme of Neutron-Rich Lead Isotopes: The Role of Effective ThreeBody Forces, Phys. Rev. Lett. 109, 162502 (2012).

[2] G. Benzoni et al., First Measurement of Beta Decay HalfLives in Neutron-Rich Tl and Bi Isotopes, Phys. Lett. B 715, 293 (2012).

[3] A. I. Morales et al., $\beta$-Decay Studies of Neutron-Rich $\mathrm{Tl}, \mathrm{Pb}$, and Bi Isotopes, Phys. Rev. C 89, 014324 (2014).

[4] A. Gottardo et al., Isomeric Decay Spectroscopy of the ${ }^{217} \mathrm{Bi}$ Isotope, Phys. Rev. C 90, 034317 (2014).

[5] L. P. Gaffney et al., Studies of Pear-Shaped Nuclei Using Accelerated Radioactive Beams, Nature (London) 497, 199 (2013).

[6] Y. Blumenfeld, T. Nilsson, and P. Van Duppen, Facilities and Methods for Radioactive Ion Beam Production, Phys. Scr. T152, 014023 (2013).

[7] B. Jonson and A. Richter, More Than Three Decades of ISOLDE Physics, Hyperfine Interact. 129, 1 (2000).

[8] V. N. Fedosseev, Yu. Kudryavtsev, and V. I. Mishin, Resonance Laser Ionization of Atoms for Nuclear Physics, Phys. Scr. 85, 058104 (2012).
[9] J. Kurpeta et al., The Decay of the Neutron-Rich Nucleus ${ }^{216}$ Bi, Eur. Phys. J. A 7, 49 (2000).

[10] J. Kurpeta et al., Isomeric and Ground-State Decay of ${ }^{215} \mathrm{Bi}$, Eur. Phys. J. A 18, 31 (2003).

[11] J. Kurpeta et al., The Decay of the New Neutron-Rich Isotope ${ }^{217} \mathrm{Bi}$, Eur. Phys. J. A 18, 5 (2003).

[12] H. De Witte, S. Eeckhaudt et al., $\beta^{-}$Decay of the NeutronRich Isotope ${ }^{215} \mathrm{~Pb}$, Phys. Rev. C 87, 067303 (2013).

[13] H. De Witte et al., First Observation of the $\beta$ Decay of Neutron-Rich ${ }^{218} \mathrm{Bi}$ by the Pulsed-Release Technique and Resonant Laser Ionization, Phys. Rev. C 69, 044305 (2004).

[14] H. De Witte et al., Nuclear Charge Radii of Neutron Deficient Lead Isotopes beyond $N=104$ Mid-shell Investigated by In-Source Laser Spectroscopy, Phys. Rev. Lett. 98, 112502 (2007).

[15] A. N. Andreyev, J. Elseviers et al., New Type of Asymmetric Fission in Proton-Rich Nuclei, Phys. Rev. Lett. 105, 252502 (2010).

[16] T. E. Cocolios, W. Dexters, M. D. Seliverstov et al., Early Onset of Ground State Deformation in Neutron Deficient Polonium Isotopes, Phys. Rev. Lett. 106, 052503 (2011).

[17] L. Ghys et al., Triple-Humped Fission-Fragment Mass Distributions in the Neutron-Deficient Lead Region, Phys. Rev. C 90, 041301(R) (2014).

[18] M. Wada, Genealogy of Gas Cells for Low-Energy RI-Beam Production, Nucl. Instrum. Methods Phys. Res., Sect. B 317, 450 (2013).

[19] R. Ferrer et al., In Gas Laser Ionization and Spectroscopy Experiments at the Superconducting Separator Spectrometer $\left(S^{3}\right)$ : Conceptual Studies and Preliminary Design, Nucl. Instrum. Methods Phys. Res., Sect. B 317, 570 (2013).

[20] S. Schwarz et al., The NSCL Cyclotron Gas Stopper-Under Construction, Nucl. Instrum. Methods Phys. Res., Sect. B 317, 463 (2013).

[21] V. N. Fedosseev, G. Huber, U. Köster, J. Lettry, V. I. Mishin, H. Ravn, and V. Sebastian, The ISOLDE Laser Ion Source for Exotic Nuclei, Hyperfine Interact. 127, 409 (2000).

[22] F. Schwellnus et al., Study of Low Work Function Materials for Hot Cavity Resonance Ionization Laser Ion Sources, Nucl. Instrum. Methods Phys. Res., Sect. B 267, 1856 (2009).

[23] P. Van Duppen et al., A New Pulsed Release Method for Element Selective Production of Neutron-Rich Isotopes near ${ }^{208} \mathrm{~Pb}$, Nucl. Instrum. Methods Phys. Res., Sect. B 134, 267 (1998).

[24] K. Blaum, C. Geppert, H.-J. Kluge, M. Mukherjee, S. Schwarz, and K. Wendt, A Novel Scheme for a Highly Selective Laser Ion Source, Nucl. Instrum. Methods Phys. Res., Sect. B 204, 331 (2003).

[25] K. Wendt, K. Blaum, K. Brück, Ch. Geppert, H.-J. Kluge, M. Mukherjee, G. Passler, S. Schwarz, S. Sirotzki, and K. Wies, A Highly Selective Laser Ion Source for Bunched, Low Emittance Beam Release, Nucl. Phys. A746, 47 (2004).

[26] K. Wies, C. Geppert, K. Blaum, K. Brück, H.-J. Kluge, S. Schwarz, and K. Wendt, Development Towards a Laser Ion Source Trap for the Production of Exotic Species, Hyperfine Interact. 162, 29 (2005). 
[27] T. Sonoda, T. E. Cocolios, J. Gentens, M. Huyse, O. Ivanov, Yu. Kudryavtsev, D. Pauwels, P. Van den Bergh, and P. Van Duppen, The Laser Ion Source Trap (LIST) Coupled to a Gas Cell Catcher, Nucl. Instrum. Methods Phys. Res., Sect. B 267, 2918 (2009).

[28] M. Reponen, I. D. Moore, I. Pohjalainen, T. Kessler, P. Karvonen, J. Kurpeta, B. Marsh, S. Piszczek, V. Sonnenschein, and J. Äystö, Gas Jet Studies Towards an Optimization of the IGISOL LIST Method, Nucl. Instrum. Methods Phys. Res., Sect. A 635, 24 (2011).

[29] I. D. Moore et al., Towards Commissioning the New IGISOL-4 Facility, Nucl. Instrum. Methods Phys. Res., Sect. B 317, 208 (2013).

[30] F. Schwellnus et al., The Laser Ion Source Trap for Highest Isobaric Selectivity in Online Exotic Isotope Production, Rev. Sci. Instrum. 81, 02A515 (2010).

[31] D. A. Fink, S. D. Richter et al., On-Line Implementation and First Operation of the Laser Ion Source and Trap at ISOLDE, Nucl. Instrum. Methods Phys. Res., Sect. B 344, 83 (2015).

[32] J. P. Lavoie, P. Bricault, J. Lassen, and M. R. Pearson, Segmented Linear Radiofrequency Quadrupole/Laser Ion Source Project at TRIUMF, Hyperfine Interact. 174, 33 (2007).

[33] S. Raeder, H. Heggen, J. Lassen, F. Ames, D. Bishop, P. Bricault, P. Kunz, A. Mjøs, and A. Teigelhöfer, An Ion Guide Laser Ion Source for Isobar-Suppressed Rare Isotope Beams, Rev. Sci. Instrum. 85, 033309 (2014).

[34] A. T. Gallant, M. Brodeur et al., Breakdown of the Isobaric Multiplet Mass Equation for the $A=20$ and 21 Multiplets, Phys. Rev. Lett. 113, 082501 (2014).

[35] B.E. Schultz, M. Brodeur et al., Precision $Q_{\mathrm{EC}}$-Value Measurement of ${ }^{23} \mathrm{Mg}$ for Testing the Cabibbo-KobayashiMaskawa Matrix Unitarity, Phys. Rev. C 90, 012501(R) (2014).

[36] D. A. Fink, S. D. Richter et al., First Application of the Laser Ion Source and Trap (LIST) for On-Line Experiments at ISOLDE, Nucl. Instrum. Methods Phys. Res., Sect. B 317, 417 (2013).

[37] I. Budinčević et al., Laser Spectroscopy of Francium Isotopes at the Borders of the Region of Reflection Asymmetry, Phys. Rev. C 90, 014317 (2014).

[38] M. D. Seliverstov, T.E. Cocolios et al., Electromagnetic Moments of Odd-A ${ }^{191-203,211}$ Po Isotopes, Phys. Rev. C 89, 034323 (2014).

[39] B. A. Marsh et al., New Developments of the In-Source Spectroscopy Method at RILIS/ISOLDE, Nucl. Instrum. Methods Phys. Res., Sect. B 317, 550 (2013).

[40] T. E. Cocolios, B. A. Marsh et al., Resonant Laser Ionization of Polonium at RILIS-ISOLDE for the Study of Groundand Isomer-State Properties, Nucl. Instrum. Methods Phys. Res., Sect. B 266, 4403 (2008).

[41] S. Rothe, V. N. Fedosseev, T. Kron, B. A. Marsh, R. E. Rossel, and K. D. A. Wendt, Narrow Linewidth Operation of the RILIS Titanium Sapphire Laser at ISOLDE/CERN, Nucl. Instrum. Methods Phys. Res., Sect. B 317, 561 (2013).

[42] B. Lommel, W. Hartmann, B. Kindler, J. Klemm, and J. Steiner, Preparation of Self-Supporting Carbon Thin Films, Nucl. Instrum. Methods Phys. Res., Sect. A 480, 199 (2002).
[43] J. Elseviers et al., $\beta$-Delayed Fission of ${ }^{180} \mathrm{Tl}$, Phys. Rev. C 88, 044321 (2013).

[44] H. De Witte et al., Alpha-Decay of Neutron-Deficient ${ }^{200} \mathrm{Fr}$ and Heavier Neighbours, Eur. Phys. J. A 23, 243 (2005).

[45] S. Kreim et al., Competition between Pairing Correlations and Deformation from the Odd-Even Mass Staggering of Francium and Radium Isotopes, Phys. Rev. C 90, 024301 (2014).

[46] S. Lukić, F. Gevaert, A. Kelić, M. V. Ricciardi, K.-H. Schmidt, and O. Yordanov, Systematic Comparison of ISOLDE-SC Yields with Calculated In-Target Production Rates, Nucl. Instrum. Methods Phys. Res., Sect. A 565, 784 (2006).

[47] A. K. Jain and B. Singh, Nuclear Data Sheets for $A=218$, Nucl. Data Sheets 107, 1027 (2006).

[48] J. Lettry, R. Catherall, P. Drumm, P. Van Duppen, A. H. M. Evensen, G. J. Focker, A. Jokinen, O. C. Jonsson, E. Kugler, and H. Ravn, Pulse Shape of the ISOLDE Radioactive Ion Beam, Nucl. Instrum. Methods Phys. Res., Sect. B 126, 130 (1997).

[49] E. Browne, Nuclear Data Sheets for $A=212$, Nucl. Data Sheets 104, 427 (2005).

[50] S.-C. Wu, Nuclear Data Sheets for $A=216$, Nucl. Data Sheets 108, 1057 (2007).

[51] Y. A. Akovali, Nuclear Data Sheets for $A=217$, Nucl. Data Sheets 100, 141 (2003).

[52] H. Jeppesen et al., Search for Beta-Delayed Charged Particles from the Halo Nucleus ${ }^{14} \mathrm{Be}$, Nucl. Phys. A709, 119 (2002).

[53] R. E. Rossel, V. N. Fedosseev, B. A. Marsh, D. Richter, S. Rothe, and K. D. A. Wendt, Data Acquisition, Remote Control and Equipment Monitoring for ISOLDE RILIS, Nucl. Instrum. Methods Phys. Res., Sect. B 317, 557 (2013).

[54] B. Cheal and K. T. Flanagan, Progress in Laser Spectroscopy at Radioactive Ion Beam Facilities, J. Phys. G 37, 113101 (2010).

[55] K. Blaum, J. Dilling, and W. Nörterhäuser, Precision Atomic Physics Techniques for Nuclear Physics with Radioactive Beams, Phys. Scr. T152, 014017 (2013).

[56] C. F. Liang, P. Paris, and R. K. Sheline, Level Structure of ${ }^{217} \mathrm{Po}$ and the Spin of the Previously Proposed ${ }^{213} \mathrm{~Pb}$ Ground State, Phys. Rev. C 56, 2324 (1997).

[57] C. F. Liang, P. Paris, R. K. Sheline, and P. Alexa, Alpha Decay of ${ }^{221} \mathrm{Rn}$ and ${ }^{217} \mathrm{Po}$; Level Structure of ${ }^{217} \mathrm{Po}$ and the ${ }^{213} \mathrm{~Pb}$ Ground State, Cesk. Cas. Fyz. 54, 189 (2004).

[58] M. D. Seliverstov, T. E. Cocolios et al., Charge Radii of Odd-A ${ }^{191-211}$ Po Isotopes, Phys. Lett. B 719, 362 (2013).

[59] B. Cheal, T. E. Cocolios, and S. Fritzsche, Laser Spectroscopy of Radioactive Isotopes: Role and Limitations of Accurate Isotope-Shift Calculations, Phys. Rev. A 86, 042501 (2012).

[60] D. Kowalewska, W. Bekk, S. Göring, A. Hanser, W. Kälber, G. Meisel, and H. Rebel, Isotope Shifts and Hyperfine Structure in Polonium Isotopes by Atomic-Beam Laser Spectroscopy, Phys. Rev. A 44, R1442 (1991).

[61] W. D. Myers and K. H. Schmidt, An Update on DropletModel Charge Distributions, Nucl. Phys. A410, 61 (1983). 
[62] D. Berdichevsky and F. Tondeur, Nuclear Core Densities, Isotope Shifts, and the Parametrization of the Droplet Model, Z. Phys. A 322, 141 (1985).

[63] P. M. Goddard, P. D. Stevenson, and A. Rios, Charge Radius Isotope Shift across the $N=126$ Shell Gap, Phys. Rev. Lett. 110, 032503 (2013).

[64] W. Borchers, R. Neugart, E. W. Otten, H. T. Duong, G. Ulm, and K. Wendt, Hyperfine Structure and Isotope Shift Investigations in ${ }^{202-222} \mathrm{Rn}$ for the Study of Nuclear Structure beyond $Z=82$, Hyperfine Interact. 34, 25 (1987).

[65] A. Coc et al., Isotope Shifts, Spins and Hyperfine Structures of ${ }^{118,146} \mathrm{Cs}$ and of Some Francium Isotopes, Nucl. Phys. A468, 1 (1986).

[66] K. Wendt, S. A. Ahmad, W. Klempt, R. Neugart, E. W. Otten, and H. H. Stroke, On the Hyperfine Structure and Isotope Shift of Radium, Z. Phys. D 4, 227 (1987).

[67] P. A. Butler and W. Nazarewicz, Intrinsic Reflection Asymmetry in Atomic Nuclei, Rev. Mod. Phys. 68, 349 (1996).

[68] M. D. Seliverstov et al., Charge Radii and Magnetic Moments of Odd-A ${ }^{183-189} \mathrm{~Pb}$ Isotopes, Eur. Phys. J. A 41, 315 (2009).

[69] A. Coc et al., Hyperfine Structures and Isotope Shifts of ${ }^{207-213,220-228}$ Fr; Possible Evidence of Octupolar Deformation, Phys. Lett. 163B, 66 (1985).

[70] M. Anselments, W. Faubel, S. Göring, A. Hanser, G. Meisel, H. Rebel, and G. Schatz, The Odd-Even Staggering of the Nuclear Charge Radii of Pb Isotopes, Nucl. Phys. A451, 471 (1986).

[71] U. Dinger, J. Eberz, G. Huber, R. Menges, S. Schröder, R. Kirchner, O. Klepper, T. Kühl, D. Marx, and G. D. Sprouse,
Nuclear Moments and Change in the Charge Radii of Neutron Deficient Lead Isotopes, Z. Phys. A 328, 253 (1987).

[72] S. B. Dutta, R. Kirchner, O. Klepper, T. U. Kühl, D. Marx, G. D. Sprouse, R. Menges, U. Dinger, G. Huber, and S. Schröder, Measurement of the Isotope Shift and Hyperfine Splitting of ${ }^{190,191,193,197} \mathrm{~Pb}$ Isotopes by Collinear Laser Spectroscopy, Z. Phys. A 341, 39 (1991).

[73] G. Fricke and K. Heilig, Nuclear Charge Radii (Springer, New York, 2004).

[74] G. Neyens, Nuclear Magnetic and Quadrupole Moments for Nuclear Structure Research on Exotic Nuclei, Rep. Prog. Phys. 66, 633 (2003).

[75] R. Bauer, J. Speth, V. Klemt, P. Ring, E. Werner, and T. Yamazaki, Magnetic Multipole Moments and Transition Probabilities of Single-Particle States around ${ }^{208} \mathrm{~Pb}$, Nucl. Phys. A209, 535 (1973).

[76] E. Browne, Nuclear Data Sheets for $A=215$, Nucl. Data Sheets 93, 763 (2001).

[77] E. Browne, Nuclear Data Sheets for $A=211$, Nucl. Data Sheets 103, 183 (2004).

[78] E. K. Hyde and A. Ghiorso, The Alpha-Branching of AcK and the Presence of Astatine in Nature, Phys. Rev. 90, 267 (1953).

[79] J. O. Rasmussen, Alpha-Decay Barrier Penetrabilities with an Exponential Nuclear Potential: Even-Even Nuclei, Phys. Rev. 113, 1593 (1959).

[80] S. Kreim et al., Recent Exploits of the ISOLTRAP Mass Spectrometer, Nucl. Instrum. Methods Phys. Res., Sect. B 317, 492 (2013). 